\title{
Precise Dimming and Color Control of Light- Emitting Diode Systems based on Color Mixing
}

\author{
Albert Lee, Member, IEEE, Huanting Chen, Member, IEEE, Siew-Chong Tan ${ }^{1}$, Senior Member, IEEE, \\ S. Y. (Ron) Hui ${ }^{2}$, Fellow, IEEE
}

\begin{abstract}
This paper proposes a closed-loop nonlinear method for precisely controlling the luminosity and correlated color temperature (CCT) of a bi-color adjustable light-emitting diode (LED) lamp. The objective is to achieve a precise and fully-independent dimming and CCT control of the light mixture emitted from a two-string LED lamp comprising warm-white and cool-white color LEDs, regardless of the operating conditions and throughout the long operating lifetime of the LED lamp. This control is formulated using the non-linear empirical LED model of the bi-color LED system. Experimental results show that with the proposed closedloop nonlinear control, both CCT and dimming control of the bi-color lamp is significantly more accurate and robust to ambient temperature variations, ambient light interference, and LED aging than the conventional linear control used in existing products. The maximum error in luminous flux employing the proposed nonlinear control method is $3 \%$, compared with $20 \%$ using the closed-loop linear method. The maximum deviation in CCT is only $1.78 \%$, compared with $27.5 \%$ with its linear counterpart.
\end{abstract}

\footnotetext{
${ }^{1}$ For future correspondence, please email: sctan@eee.hku.hk.

${ }^{2}$ The authors are with Department of Electrical and Electronic Engineering, The University of Hong Kong, Hong Kong. S. Y. R. Hui is also with Department of Electrical and Electronic Engineering, Imperial College London, U.K.
} 


\section{Index Terms}

Bi-color LED lamp, closed-loop nonlinear control, correlated color temperature (CCT), dimming, lightemitting diodes (LED), light control.

\section{NOMENCLATURE}

$D_{w} \quad$ Duty ratio for warm-white LED

$D_{c} \quad$ Duty ratio for cool-white LED

$\Delta D_{O S} \quad$ Duty ratio offset

$\phi \quad$ Luminous flux (lm)

$\phi_{T} \quad$ Target luminous flux (lm)

$\phi_{M} \quad$ Combined luminous flux of cool-white and warm-white LEDs (lm)

$\phi_{c} \quad$ Luminous flux of cool-white LEDs (lm)

$\phi_{w} \quad$ Luminous flux of warm-white LEDs (lm)

CCT Correlated color temperature (K)

$C C T_{T} \quad$ Target correlated color temperature (K)

$C C T_{M}$ Combined correlated color temperature of cool-white and warm-white LEDs (K)

$C C T_{c} \quad$ Correlated color temperature of cool-white LEDs (K)

$C C T_{w} \quad$ Correleted color temperature of warm-white LEDs (K)

RGB Red, green, and blue values of the color sensor

XYZ CIE tri-stimulus values

$(x, y, z) \quad$ Chromaticity coordinates

\section{INTRODUCTION}

High-power light-emitting diode (LED) has the conspicuous advantages of high luminous efficacy, reduced energy consumption, more environmentally friendly, and potentially longer operating lifetime over the conventional fluorescent and incandescent lamps for general lighting applications. A major challenge facing the lighting industry is the need for color consistency in LED illumination [1]-[6]. Due to aging and changing operating conditions, even LEDs from the same bin supplied by a given manufacturer can deliver inconsistent color temperature over time. Most notably, variations in operating temperature can lead to noticeable differences in color appearance [2], [7], [8]. More recently, researchers and lighting vendors are gearing towards a two-string LED system consisting of warm white and cool white LEDs (also referred to as a bi-color LED lamp), which enables deep dimming and wide CCT adjustment of their emitted light mixture [9]-[12].

Dimmable bi-color LED lamps with adjustable CCT are emerging and value-added products in the general lighting market, and are also found in high-end lighting applications, such as studio lightings, hotel and museum ceiling lightings, and photography applications. Recent breakthroughs in LED technology enable 
LEDs to be adopted across an even wider range of practical applications including surgical lighting systems (SLS), photolithography room lighting, and anti-glare downlight for optimal indoor lighting systems [13]-[18]. However, all of such products are based on simplistic linear control methodologies that disregard the actual nonlinear characteristic ${ }^{3}$ of the LEDs. Therefore, while they achieve the basic functionalities of dimming and CCT control, the achievable light control is non-independent and highly inaccurate [9]-[12].

LED systems involve highly complex interactions of power, heat, light and color. An example of such complex interdependency is found when a change of LED power causes corresponding a change of light intensity and junction temperature, which in turn causes a shift of color spectrum. Existing linear approach adopted in bi-color LED lamps assumes that the nominal color temperatures of the warm-white and cool-white LEDs remain constant. It employs duty cycle control to mix color. Such assumption obviously fails to account for the temperature dependent color temperature of LED and is the main reason for their inability in providing precise dimming and color control.

To resolve this issue, a non-linear empirical LED model of the bi-color LED system useful for attaining high-quality and wide-range dimming and CCT control of the system has been recently proposed [19]. The work in [19] entails the fundamental theory and approach of developing such an LED model, which is an important component towards achieving a high-quality bi-color LED system. Building upon that, this paper presents a closed-loop nonlinear control method which achieves a truly robust, precise, and independent control of the luminous flux and CCT of the bi-color LED system, regardless of the operating conditions and throughout the long operating lifetime of the LED lamp, as seen in practical LED systems.

The proposed LED control system involves an underlying nonlinear feedback control scheme, a low-cost controller, a low-cost, low-power RGB color sensor that obtains real-time feedback of CCT and luminous flux values, and a two-string LED lamp. Experimental results are included to confirm that the proposed nonlinear method can provide significantly more precise CCT and dimming control of the bi-color lamp than exiting

\footnotetext{
${ }^{3}$ The overall brightness and CCT of the combined light mixture emitted from the bi-color LEDs are highly nonlinear coupled functions of the operating temperature, current amplitude, drive technique, and heatsink size on the light intensity and CCT [2], [19]-[22].
} 
linear method even under ambient temperature variations, ambient light interference. Moreover, due to the feedback mechanism present in the proposed control scheme, which ensures that the scheme only reacts to the actual combined measured output of the LEDs, a constrained variation of the optical and electrical characteristics of LEDs across different bins within the lamp will not affect the accuracy of the controlled performance achievable with the proposed control. Therefore, the use of LEDs with variations in optical and electrical properties due to binning is possible with the proposed scheme. The generation of precise and consistent light output from the proposed bi-color LED system opens up new opportunities for achieving highquality dimming and color-mixing in high-end lighting applications.

\section{PROPOSED CLOSED-LOOP NONLINEAR CONTROL SCHEME}

A. Characteristic of an Independent Dimming and CCT Control

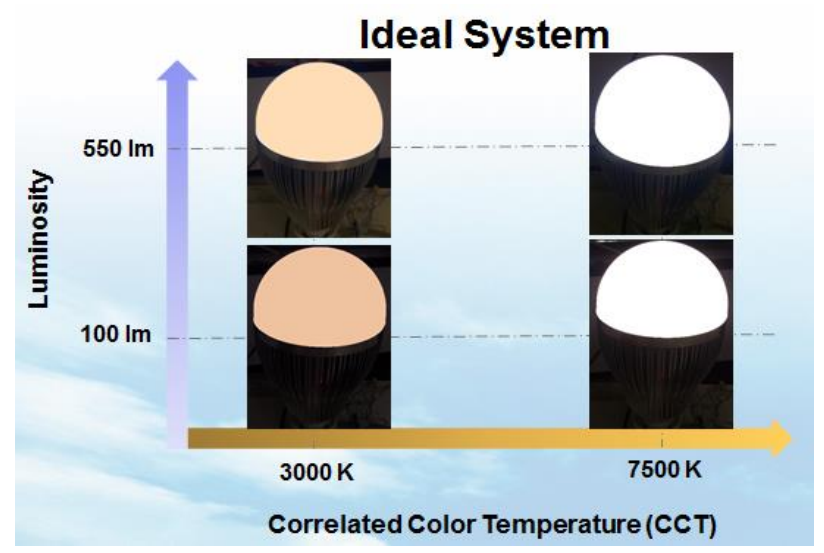

Fig. 1. An ideal bi-color LED system with independent light intensity and color control.

Fig. 1 gives a graphical illustration of the ideal "bi-color LED system" concept with independent (or decoupled) light intensity and color control. The bi-color LED lamp consists of warm-white and cool-white LEDs. A typical white LED is made up of a blue LED chip and is coated with yellow phosphor. A bi-color LED lamp is said to be ideal when a change in the CCT does not in any way affect the luminous flux. Likewise, the CCT remains unchanged despite a change in the luminous flux. However, due to the highly nonlinear and coupled relationships between light intensity, CCT, driving current, temperature of the LEDs, it is not 
straightforward to achieve precise dimming and color control with common linear feedback control. Moreover, variations in ambient temperature, ambient light intensity and LED aging will lead to even more substantial deviations of the actual flux and CCT of the lamp from the desired target flux and CCT. A CCT deviation of greater than $\pm 200 \mathrm{~K}$, of which the color drift is easily detectable by human eyes, is common in existing bicolor LED lamps.

\section{B. Operating Principle}

Fig. 2 shows the functional block diagram of the proposed system. Unlike its open-loop counterpart presented in [19], a color sensor is employed in the closed-loop system to continuously monitor the effective light output from the two LED sources, each of which emits light with a different CCT. The color sensor provides a digital return of the red, green, blue (RGB), and clear light sensing values to the microcontroller (MCU) via the standard two-wire $\mathrm{I}^{2} \mathrm{C}$ serial bus.

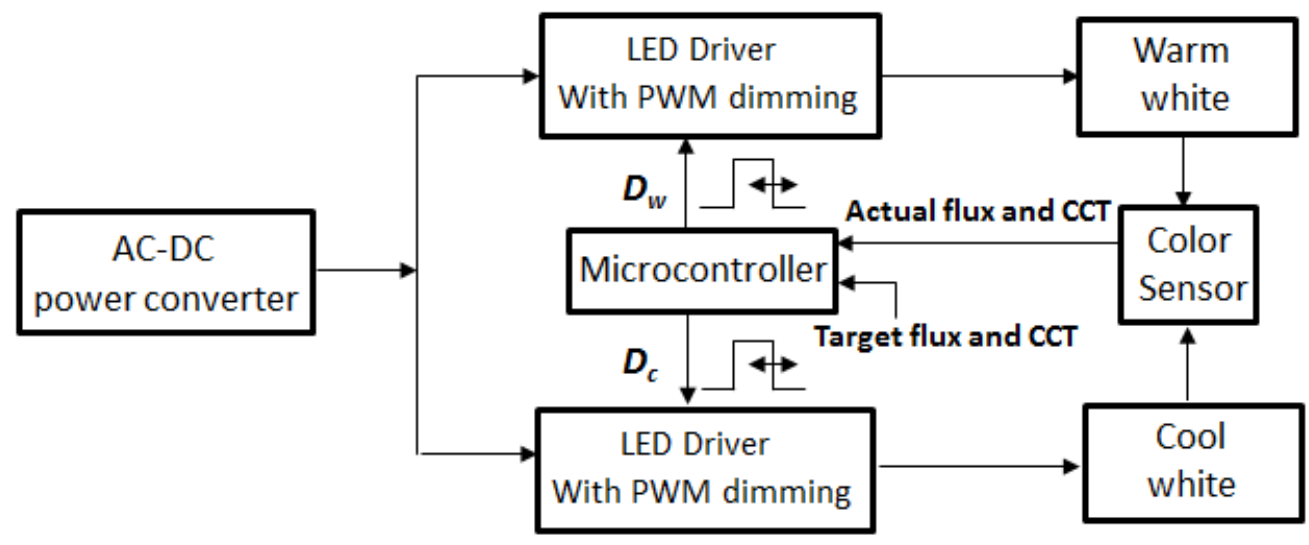

Fig. 2. Functional block diagram of the proposed closed-loop bi-color LED system.

The power stage of the bi-color LED system comprises an AC-DC power converter which converts an AC line voltage of $240 \mathrm{~V}$ to a DC voltage of $48 \mathrm{~V}$. The AC-DC power converter is connected to a pair of constantcurrent buck regulators (i.e. LED drivers), one of which drives the warm-white LED string and another drives the cool-white LED string. LED dimming is performed by using pulse-width modulation (PWM). The MCU generates two distinct PWM signals with a particular set of duty ratios $\left(D_{w}, D_{c}\right)$ to independently adjust the 
average currents for the warm-white and cool-white LED strings. A calibrated color sensor embedded on the LED printed circuit board (PCB) is used to periodically measure the combined luminous flux and CCT from both strings. The actual values of flux and CCT are then fed back to the MCU which are compared against the target values of flux and CCT. Using a dedicated software algorithm, the MCU determines a new set of PWM duty ratios for driving the two LED strings in such a way that the actual values of flux and CCT continue to track closely their corresponding target values regardless of changes in the operating environment. This forms the basis of the proposed closed-loop LED system.

In principle, the duty ratios $\left(D_{w}, D_{c}\right)$ necessary to generate specific flux and CCT values can be derived analytically using the nonlinear empirical luminous and CCT models [19], [23]. This nonlinear model takes into account the thermal interdependency of the two color sources. Nonetheless, it is impractical to determine a complete set of duty ratios for all possible flux and CCT values during run-time because of the computational complexity and huge demand for memory storage which can readily exceed the capacity of a low-cost microcontroller. Instead, a look-up table (LUT) is created which consists of the duty ratios for a finite set of the required flux and CCT values. The size of the LUT is small enough so that it can easily be saved in flash program memory of the MCU, thereby easing the actual implementation. By performing piecewise linear interpolation using the two closest data points from the LUT, the corrected duty ratios for the target flux and CCT values can be obtained. The following section provides a theoretical analysis of the underlying flux and CCT control methods.

\section{Theoretical Consideration}

According to the nonlinear model of a bi-color white LED lamp [19], the combined luminous flux $\phi_{M}$ from both the warm-white and cool-white LEDs can be expressed in the general form as

$$
\phi_{M}\left(D_{c}, D_{w}\right)=\phi_{c}\left(D_{c}, D_{w}\right)+\phi_{W}\left(D_{c}, D_{w}\right)=\left(\phi_{c 0}+\phi_{w 0}\right)-\alpha_{c} e^{-\left(\beta_{c} D_{w}+\beta_{c, \min }\right) D_{c}}-\alpha_{w} e^{-\left(\beta_{w} D_{c}+\beta_{w, \min }\right) D_{w}}
$$

where $\phi_{c 0}, \phi_{w 0}, \alpha_{c}, \alpha_{\mathrm{w}}, \beta_{c}, \beta_{w}, \beta_{c, \min }$, and $\beta_{w, \min }$ are positive constants whose values are extracted from the experimental results using curve-fitting techniques [19]. $D_{c}$ and $D_{w}$ are the programmable duty ratio for the cool-white and warm-white LEDs, respectively, and are defined as $0 \leq D_{c} \leq 1$ and $0 \leq D_{w} \leq 1$. Note that the 
individual flux from cool-white (or warm-white) LED is a function of both $D_{c}$ and $D_{w}$ which accounts for the thermal interdependency effect of the two color sources. From Equation (1), the total luminous flux can be increased (or decreased) with a larger (or smaller) duty ratio of $\left(D_{c}, D_{w}\right)$. An increased PWM duty ratio raises the average current across the LED which increases its brightness. Likewise, a smaller PWM duty ratio reduces the average current across the LED which reduces its brightness.

A typical digital color sensor returns the red $(\mathrm{R})$, green $(\mathrm{G})$, blue $(\mathrm{B})$ data which can be used to determine the CCT of a given light source. As a first step, the sensor response (RGB) must be mapped to the $\mathrm{CIE}^{4}$ tristimulus values (XYZ). The chromaticity coordinates $(x, y)$ can then be calculated and hence, CCT can be obtained using McCamy's formula. Fig. 3 gives an overview of the computation process for CCT [24].

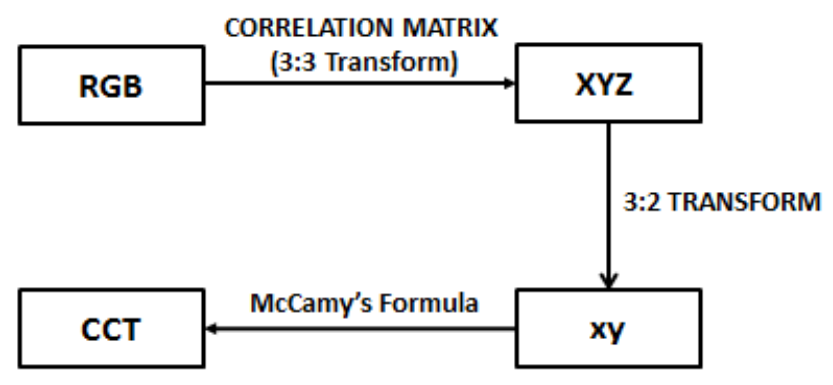

Fig. 3. Overview of the CCT computation process.

Mathematically, the tri-stimulus values (XYZ) in the CIE color space can be expressed in terms of the RGB values of a light source and a $3 \times 3$ Correlation Matrix.

$$
\left[\begin{array}{l}
X \\
Y \\
Z
\end{array}\right]=\left[\begin{array}{lll}
a_{11} & a_{12} & a_{13} \\
a_{21} & a_{22} & a_{23} \\
a_{31} & a_{32} & a_{33}
\end{array}\right]\left[\begin{array}{l}
R \\
G \\
B
\end{array}\right]
$$

where $C=\left[\begin{array}{lll}a_{11} & a_{12} & a_{13} \\ a_{21} & a_{22} & a_{23} \\ a_{31} & a_{32} & a_{33}\end{array}\right]$ is the Correlation Matrix.

Once the tri-stimulus values are determined, the chromaticity coordinates can be readily obtained as follows.

\footnotetext{
${ }^{4} \mathrm{CIE}$ stands for Commission Internationale de l'Eclairage which is the main international organization concerned with color and color measurement.
} 


$$
\left\{\begin{array}{l}
x=\frac{X}{X+Y+Z} \\
y=\frac{Y}{X+Y+Z} \\
z=\frac{Z}{X+Y+Z}
\end{array}\right.
$$

Using McCamy's formula, the CCT value can be obtained from the chromaticity coordinates [24].

$$
C C T=449 n^{3}+3525 n^{2}+6823.3 n+5520.33
$$

where $n=\frac{(x-0.3320)}{(0.1858-y)}$

In light science, the actual CCT value measured by the color sensor can be verified against the target CCT value calibrated by the integrating sphere. In order to obtain the coefficients in the correlation matrix defined in (2), three rounds of light measurement are necessary. For the first set of measurement, only the warm-white LEDs are turned on. The $\left(X_{w} Y_{w} Z_{w}\right)$ tri-stimulus values can be obtained from the integrating sphere and the $\left(R_{w} G_{w} B_{w}\right)$ signals from the colorimeter. The second measurement is similar to the first one in which only the cool-white LEDs are turned on. The corresponding values of $\left(X_{c} Y_{c} Z_{c}\right)$ and $\left(R_{c} G_{c} B_{c}\right)$ are obtained. For the third measurement, both warm-white and cool-white LEDs are turned on such that the combined CCT lies somewhere in the middle range. Again, the corresponding values of $\left(X_{n} Y_{n} Z_{n}\right)$ and $\left(R_{n} G_{n} B_{n}\right)$ are obtained. Hence, a tri-stimulus matrix is defined as

$$
T=\left[\begin{array}{ccc}
X_{w} & X_{c} & X_{n} \\
Y_{w} & Y_{c} & Y_{n} \\
Z_{w} & Z_{c} & Z_{n}
\end{array}\right]
$$

Likewise, the RGB values obtained from the color sensor are arranged in a matrix form known as a Sensor Response Matrix which is expressed as

$$
S=\left[\begin{array}{lll}
R_{w} & R_{c} & R_{n} \\
G_{w} & G_{c} & G_{n} \\
B_{w} & B_{c} & B_{n}
\end{array}\right]
$$


Based on (2), the Tri-stimulus Matrix $T$ can be expressed in terms of the Correlation Matrix $C$ and the Sensor Response Matrix $S$, i.e. $T=C S$. Now, we can calculate $C$ by multiplying both sides by $S^{-1}$, i.e. $C=T S^{-1}$. Hence, $C$ can be represented as

$$
C=\left[\begin{array}{lll}
a_{11} & a_{12} & a_{13} \\
a_{21} & a_{22} & a_{23} \\
a_{31} & a_{32} & a_{33}
\end{array}\right]=\left[\begin{array}{ccc}
X_{w} & X_{c} & X_{n} \\
Y_{w} & Y_{c} & Y_{n} \\
Z_{w} & Z_{c} & Z_{n}
\end{array}\right]\left[\begin{array}{lll}
R_{w} & R_{c} & R_{n} \\
G_{w} & G_{c} & G_{n} \\
B_{w} & B_{c} & B_{n}
\end{array}\right]^{-1}
$$

Once the correlation matrix is determined from (7), the actual CCT value can be calculated using equations (2)-(4) for any particular set of RGB values measured by the color sensor. In this way, the target CCT from the integrating sphere can be used to check against the actual CCT from the color sensor. Table 1 compares the target CCT and the corresponding measured CCT for the proposed bi-color white LED system consisting of 8 warm-white LEDs and 8 cool-white LEDs. The maximum percentage error is only $1.708 \%$. Basically, all the CCT errors in Table 1 fall within the maximum allowable CCT tolerances which are specified in the ANSI Standard C78.377 as given in Table 2 and are therefore non-perceivable to human eyes [25]. It is shown that the measured CCT values from the color sensor are consistent with the corresponding target values from the integrating sphere. This verifies that the color sensor can achieve high accuracy in the CCT measurements.

\begin{tabular}{|c|c|c|c|c|c|c|}
\hline $\begin{array}{c}\text { Total } \\
\text { Flux }(\mathrm{lm})\end{array}$ & $\begin{array}{l}\text { Warm-white } \\
\text { Flux (lm) }\end{array}$ & $\begin{array}{l}\text { Cool-white } \\
\text { Flux (lm) }\end{array}$ & $\begin{array}{c}\text { Target CCT (Sphere) } \\
(\mathbf{K})\end{array}$ & $\begin{array}{c}\text { Measured CCT (Sensor) } \\
(\mathbf{K})\end{array}$ & $\begin{array}{c}\text { Error } \\
\text { in } \mathbf{C C T}(\mathbf{K})\end{array}$ & $\begin{array}{l}\text { \% Error } \\
\text { in CCT }\end{array}$ \\
\hline 50 & $\begin{array}{c}50 \\
0\end{array}$ & $\begin{array}{c}0 \\
50\end{array}$ & $\begin{array}{l}2580 \\
6040\end{array}$ & $\begin{array}{l}2563.67 \\
6143.16\end{array}$ & $\begin{array}{c}16.33 \\
103.16\end{array}$ & $\begin{array}{l}0.633 \% \\
1.708 \%\end{array}$ \\
\hline 100 & $\begin{array}{c}100 \\
50 \\
0\end{array}$ & $\begin{array}{c}0 \\
50 \\
100\end{array}$ & $\begin{array}{l}2581 \\
3703 \\
6046\end{array}$ & $\begin{array}{l}2565.72 \\
3709.52 \\
6126.35\end{array}$ & $\begin{array}{c}15.28 \\
6.52 \\
80.35\end{array}$ & $\begin{array}{l}0.592 \% \\
0.176 \% \\
1.329 \%\end{array}$ \\
\hline 200 & $\begin{array}{c}200 \\
150 \\
100 \\
50 \\
0\end{array}$ & $\begin{array}{c}0 \\
50 \\
100 \\
150 \\
200\end{array}$ & $\begin{array}{l}2578 \\
3042 \\
3779 \\
4796 \\
6107\end{array}$ & $\begin{array}{l}2568.47 \\
3041.03 \\
3781.64 \\
4804.94 \\
6149.20\end{array}$ & $\begin{array}{c}9.53 \\
0.97 \\
2.64 \\
8.94 \\
42.20\end{array}$ & $\begin{array}{l}0.370 \% \\
0.032 \% \\
0.070 \% \\
0.186 \% \\
0.691 \%\end{array}$ \\
\hline 300 & $\begin{array}{c}300 \\
250 \\
200 \\
150 \\
100 \\
50 \\
0 \\
\end{array}$ & $\begin{array}{c}0 \\
50 \\
100 \\
150 \\
200 \\
250 \\
300 \\
\end{array}$ & $\begin{array}{l}2581 \\
2869 \\
3266 \\
3751 \\
4407 \\
5182 \\
6201 \\
\end{array}$ & $\begin{array}{l}2572.64 \\
2866.63 \\
3270.22 \\
3761.22 \\
4406.15 \\
5176.36 \\
6203.81 \\
\end{array}$ & $\begin{array}{c}8.36 \\
2.37 \\
4.22 \\
10.22 \\
0.85 \\
5.64 \\
2.81 \\
\end{array}$ & $\begin{array}{l}0.324 \% \\
0.083 \% \\
0.129 \% \\
0.272 \% \\
0.019 \% \\
0.109 \% \\
0.045 \%\end{array}$ \\
\hline
\end{tabular}

Table 1. Comparison of the target CCT from integration sphere and the measured CCT from the color sensor for the proposed bi-color white LED system. 


\begin{tabular}{|c|c|c|}
\hline Nominal CCT & Target CCT and Tolerance (K) & Max. Tolerance in CCT (\%) \\
\hline $2700 \mathrm{~K}$ & $2725 \pm 145$ & $\pm 5.32 \%$ \\
\hline $3000 \mathrm{~K}$ & $3045 \pm 175$ & $\pm 5.75 \%$ \\
\hline $3500 \mathrm{~K}$ & $3465 \pm 245$ & $\pm 7.07 \%$ \\
\hline $4000 \mathrm{~K}$ & $3985 \pm 275$ & $\pm 6.90 \%$ \\
\hline $4500 \mathrm{~K}$ & $4503 \pm 243$ & $\pm 5.40 \%$ \\
\hline $5000 \mathrm{~K}$ & $5028 \pm 283$ & $\pm 5.63 \%$ \\
\hline $5700 \mathrm{~K}$ & $5665 \pm 355$ & $\pm 6.27 \%$ \\
\hline $6500 \mathrm{~K}$ & $6530 \pm 510$ & $\pm 7.81 \%$ \\
\hline
\end{tabular}

Table 2. Maximum allowable CCT tolerance at each nominal CCT value (extracted from [25]).

Before performing any flux calibrations for the color sensor, it is necessary to validate the luminous flux obtained directly by our light source test system. The test system consists of an integration sphere (with a diameter of about 1.5 meters), the PMS-50 Spectrophotocolorimeter, ambient temperature detector, power supply, etc. Table 3 shows the specifications of the PMS-50 Spectrophotocolorimeter [26].

\begin{tabular}{|c|c|c|}
\hline \multirow{3}{*}{ Wavelength } & Range & $380-800 \mathrm{~nm}$ \\
\cline { 2 - 3 } & Accuracy & $\pm 0.2 \mathrm{~nm}$ \\
\cline { 2 - 3 } & Repeatability & $\pm 0.1 \mathrm{~nm}$ \\
\hline Chromaticity Coordinate & Accuracy $(\Delta \mathrm{x}, \Delta \mathrm{y})$ & \pm 0.0003 \\
\hline Spectrum Sample Interval & Normal & $1 \mathrm{~nm}$ \\
\hline \multirow{3}{*}{ Photometry } & Linearity & $\pm 0.3 \%$ \\
\cline { 2 - 3 } & Accuracy & Class 1 or standard class \\
\cline { 2 - 3 } & Range (luminous flux $)$ & $0.01 \mathrm{~lm}-1.9999 \mathrm{x} 10^{5} \mathrm{~lm}$ \\
\hline \multirow{2}{*}{ Color Temperature } & Measurement Range & $1000 \mathrm{~K}-100000 \mathrm{~K}$ \\
\cline { 2 - 3 } & Measurement Accuracy & $\pm 0.3 \%$ \\
\hline Rendering Index & Measurement Accuracy & $\pm(0.3 \% \mathrm{rdg}+0.5)$ \\
\hline Color Difference & Measurement Accuracy & $\pm 0.5 \mathrm{SDCM}$ \\
\hline Temperature & Accuracy & $\pm 0.5^{\circ} \mathrm{C}$ \\
\hline Power Source & AC Voltage & $210 \mathrm{~V}-240 \mathrm{~V}(50 / 60 \mathrm{~Hz})$ \\
\hline
\end{tabular}

Table 3. Specifications of the PMS-50 Spectrophotocolorimeter [26].

The validation of the flux measurement by our tester is accomplished by comparing its $\mathrm{Y}$ color matching function with the standard Y color matching function from CIE 1931. Fig. 4 shows that the Y color matching function from the PMS-50 color spectrometer is in good agreement with that from CIE 1931. This means that our test system can achieve highly accurate measurement of luminous flux which is regarded as an ideal or reference value for subsequent flux measurements by the color sensor. So, as long as the measured flux from 
the color sensor is consistent with the reference flux from the tester under the same lighting conditions, we can also claim that the color sensor can achieve high accuracy in flux measurements.

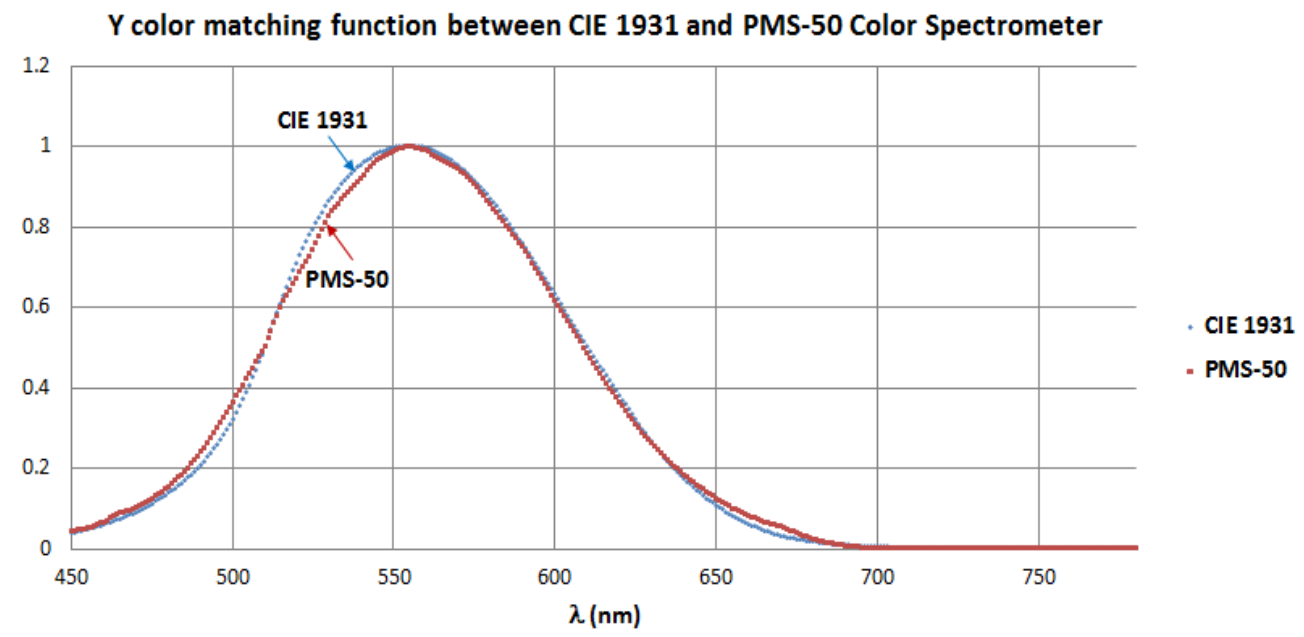

Fig. 4. Comparison of the Y color matching function from CIE 1931 with that from PMS-50 Spectrometer.

The tri-stimulus $Y$ value actually represents the illuminance (luminance) of the LED source [24]. The luminous flux $\phi$ can therefore be calculated by multiplying $Y$ by a proportionality constant $k$ which is represented as

$$
\phi=k Y
$$

The value of $k$ can be obtained from calibrating the color sensor. Based on the first set of measurement in which only the warm-white LEDs are turned on, the value of $Y$ can be calculated by using (2) as follows.

$$
Y=a_{21} R+a_{22} G+a_{23} B
$$

Since the values of $Y$ and $\phi$ are known, the proportionality constant $k$ can be calculated using (8). Therefore, the luminous flux for other measurements can be determined by multiplying $Y$ by $k$. The consistency of the values of $k$ across various flux values is also examined. Based on our actual measurements, the values of $k$ are plotted for different flux values as depicted in Fig. 5. It shows that the values of $\mathrm{k}$ are consistent across different luminous flux. The average value of $k$ is 0.1378 with a maximum deviation of 0.0038 which is reasonably small. This average value of $\mathrm{k}$ will then be used to calculate the luminous flux from any RGB values measured by the color sensor. 


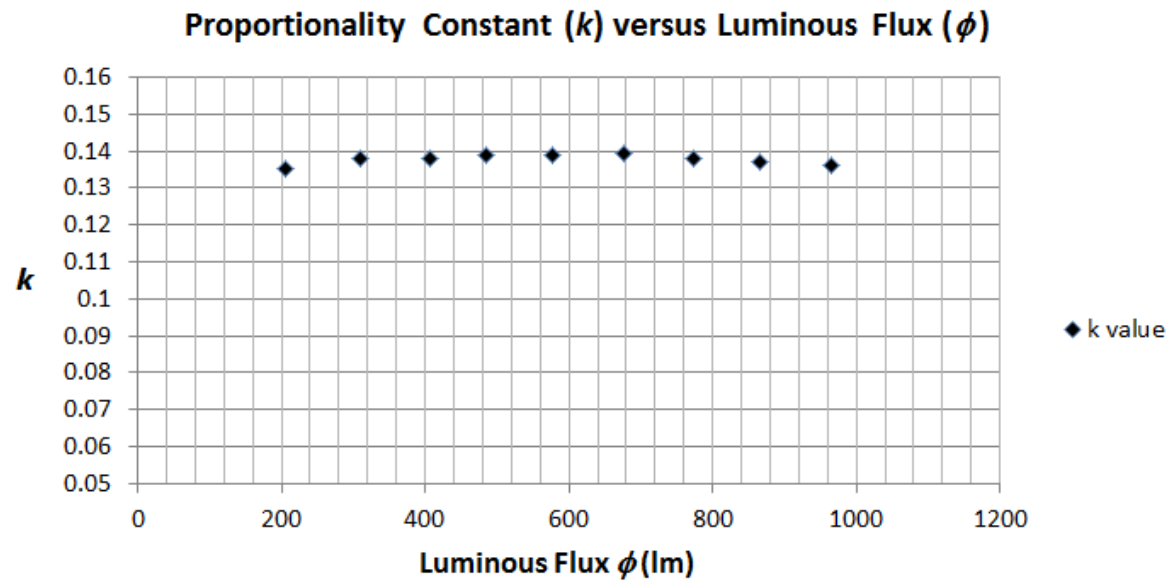

Fig. 5. The values of the proportionality constant $k$ across different luminous flux values.

The target luminous flux from the integrating sphere can be used to check against the actual luminous flux from the color sensor. Table 4 gives a comparison of the target flux and the corresponding measured flux for the proposed bi-color white LED system. The maximum percentage error is only $1.731 \%$. It shows that the measured flux values from the color sensor agree closely with the corresponding target ones from the integrating sphere. This verifies that the color sensor can achieve high accuracy in the flux measurements.

\begin{tabular}{|c|c|c|c|c|c|}
\hline $\begin{array}{c}\text { Target Flux } \\
(\mathbf{l m})\end{array}$ & $\begin{array}{c}\text { Corresponding } \\
\text { CCT (K) }\end{array}$ & $\begin{array}{c}\text { Reference Flux (Sphere) } \\
(\mathbf{l m})\end{array}$ & $\begin{array}{c}\text { Measured Flux (Sensor) } \\
(\mathbf{l m})\end{array}$ & $\begin{array}{c}\text { Error } \\
\text { in Flux (lm) }\end{array}$ & $\begin{array}{c}\text { \% Error } \\
\text { in Flux }\end{array}$ \\
\hline \multirow{2}{*}{50} & 2700 & 52.49 & 52.12 & 0.37 & 0.705 \\
& 6500 & 48.47 & 47.76 & 0.71 & 1.465 \\
\hline \multirow{3}{*}{100} & 2700 & 97.74 & 97.10 & 0.64 & 0.655 \\
& 4600 & 98.53 & 98.72 & 0.19 & 0.193 \\
& 6500 & 101.12 & 99.37 & 1.75 & 1.731 \\
\hline \multirow{3}{*}{150} & 2700 & 152.13 & 151.19 & 0.94 & 0.618 \\
& 5200 & 148.49 & 148.42 & 0.07 & 0.047 \\
& 6500 & 153.67 & 151.27 & 2.40 & 1.562 \\
\hline \multirow{2}{*}{200} & 2700 & 200.4 & 199.51 & 0.89 & 0.444 \\
& 4600 & 194.50 & 194.74 & 0.24 & 0.123 \\
& 6500 & 201.14 & 198.86 & 2.28 & 1.134 \\
\hline \multirow{2}{*}{250} & 2700 & 252.92 & 252.32 & 0.60 & 0.237 \\
& 5000 & 249.89 & 249.83 & 0.06 & 0.024 \\
& 6500 & 252.52 & 251.43 & 1.09 & 0.432 \\
\hline \multirow{2}{*}{300} & 2700 & 298.90 & 298.89 & 0.01 & 0.003 \\
& 4600 & 306.16 & 306.91 & 0.75 & 0.245 \\
\hline
\end{tabular}

Table 4. Comparison of the target flux from integration sphere and the measured flux from the color sensor for the proposed bi-color white LED system.

In general, for a bi-color white LED system, the overall tri-stimulus values of the combined light is the sum of the tri-stimulus values for warm-white LEDs $\left(X_{w} Y_{w} Z_{w}\right)$ and the tri-stimulus values for cool-white LEDs $\left(X_{c} Y_{c} Z_{c}\right)$ 
[19]. As a result, the mixed CCT of the bi-color system based on the nonlinear LED model in [19] can be expressed as

$$
C C T_{M}\left(D_{c}, D_{w}\right)=\frac{\phi_{C}\left(D_{c}, D_{w}\right)+\phi_{W}\left(D_{c}, D_{w}\right)}{\frac{\phi_{C}\left(D_{c}, D_{w}\right)}{C C T_{C, \text { avg }}\left(D_{T}\right)}+\frac{\phi_{W}\left(D_{c}, D_{w}\right)}{C C T_{W, \text { avg }}\left(D_{T}\right)}}
$$

where

$$
\begin{aligned}
& C C T_{C, \text { avg }}\left(D_{T}\right)=\frac{C C T_{C, \text { max }}-C C T_{C, \text { min }}}{D_{T, \text { max }}-D_{T, \text { min }}}\left(D_{T}-D_{T, \text { min }}\right)+C C T_{C, \text { min }} \text { and } \\
& C C T_{W, \text { avg }}\left(D_{T}\right)=\frac{C C T_{W, \text { max }}-C C T_{W, \text { min }}}{D_{T, \text { max }}-D_{T, \text { min }}}\left(D_{T}-D_{T, \text { min }}\right)+C C T_{W, \text { min }} \text { for } D_{T}=D_{c}+D_{w}
\end{aligned}
$$

Here, the term $D_{T}$ represents the total duty ratio applied and $0 \leq D_{T} \leq 2$. Thus, for a particular set of flux and CCT values, $D_{c}$ and $D_{w}$ can be numerically solved from (1) and (2). Fig. 6(a) shows a plot of the total luminous flux $\phi_{M}$ against the duty ratio of the warm-white LED $D_{w}$ for CCT values of $3300 \mathrm{~K}, 5400 \mathrm{~K}$ and $7500 \mathrm{~K}$. Fig. 6(b) shows a plot of the total luminous flux $\phi_{M}$ against the duty ratio of the cool-white LED. i.e., $D_{c}$, for CCT values of $3300 \mathrm{~K}, 5400 \mathrm{~K}$ and $7500 \mathrm{~K}$.

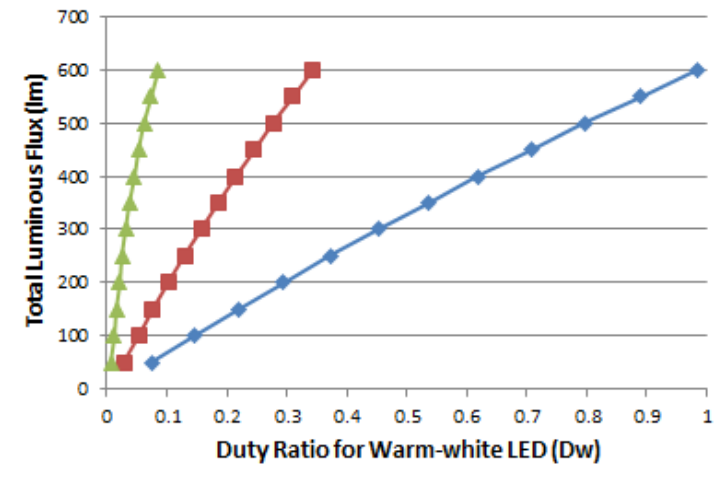

(a)

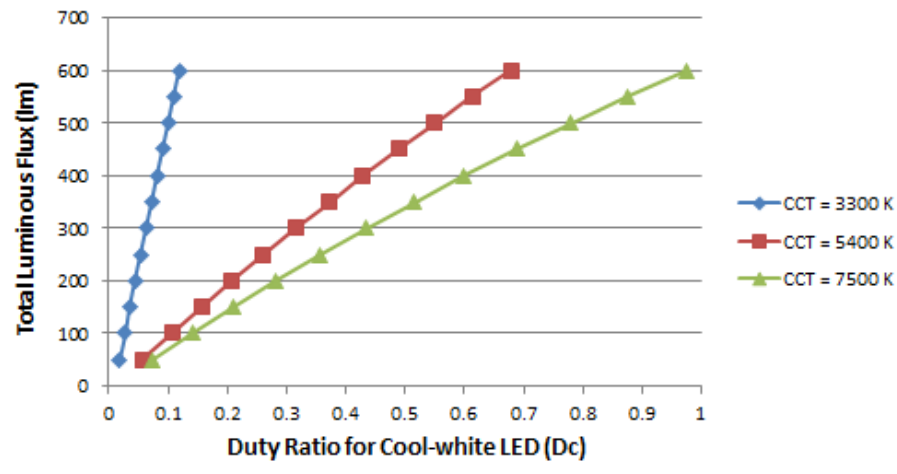

(b)

Fig. 6. Total luminous flux values versus the duty ratios for (a) warm-white LED and (b) cool-white LED.

Fig. 7a shows a plot of the effective CCT against the duty ratio of the warm-white LED, i.e., $D_{w}$, for luminous flux values of $100 \mathrm{~lm}, 300 \mathrm{~lm}$ and $550 \mathrm{~lm}$. Fig. 7b shows a plot of the effective CCT against the duty ratio of the cool-white LED, i.e., $D_{c}$, for luminous flux values of $100 \mathrm{~lm}, 300 \mathrm{~lm}$ and $550 \mathrm{~lm}$. 


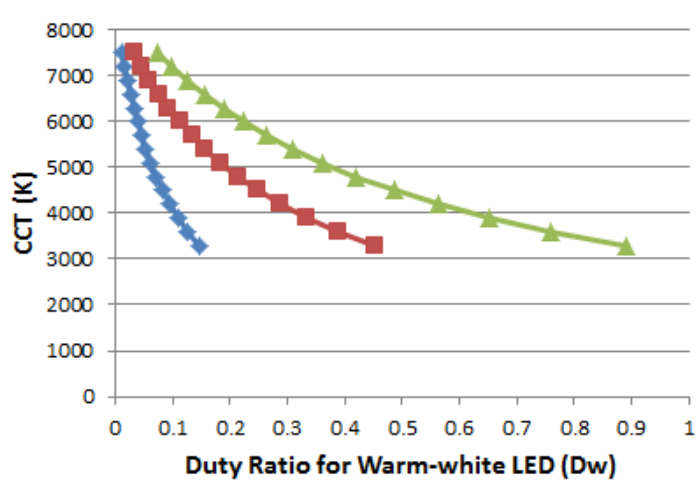

(a)

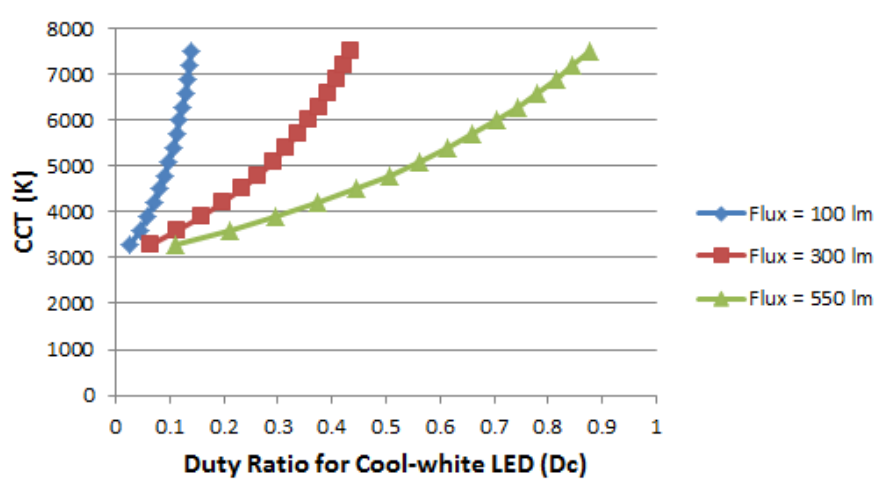

(b)

Fig. 7. Combined CCT value of bi-color LED lamp against duty ratios of (a) warm-white and (b) cool-white LED.

For a given set of duty ratios, the theoretical values of luminous flux and CCT can be predicted based on the nonlinear LED model. Nonetheless, due to variations in operating condition and LED imperfections, the actual values of flux and CCT will be different from the corresponding calculated values even when the same set of duty ratio is used. In other words, the actual flux profile will be shifted relative to the theoretical flux profile for a particular CCT value. Therefore, a closed-loop system is needed to compensate for the offset in flux and CCT. Piecewise linear interpolation is employed in the proposed closed-loop control scheme in order to estimate the duty ratio offset $\Delta D_{O S}$ which accounts for the increase (or decrease) in flux or CCT. Specifically, if the measured luminous flux $\phi_{M}$ is larger than the target flux $\phi_{T}$, the estimated duty ratio offset will be subtracted from the current values of $D_{c}$ and $D_{w}$ to provide more dimming to the LED. Likewise, if the measured flux is smaller than the target flux, the duty ratio offset is added to the current values of $D_{c}$ and $D_{w}$ to increase the luminous intensity. This is a recursive process until the system reaches the steady-state condition in which the measured flux and CCT values are in close agreement with the corresponding target values. Fig. 8 illustrates the piecewise linear interpolation scheme for determining the new duty ratio when (a) the measured flux is larger than the target flux and (b) the measured flux is smaller than the target flux. 


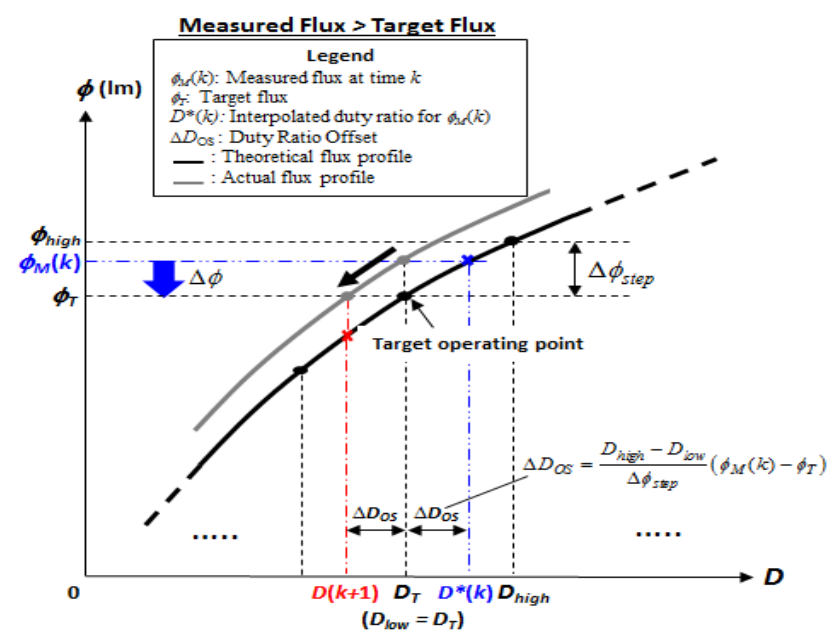

(a)

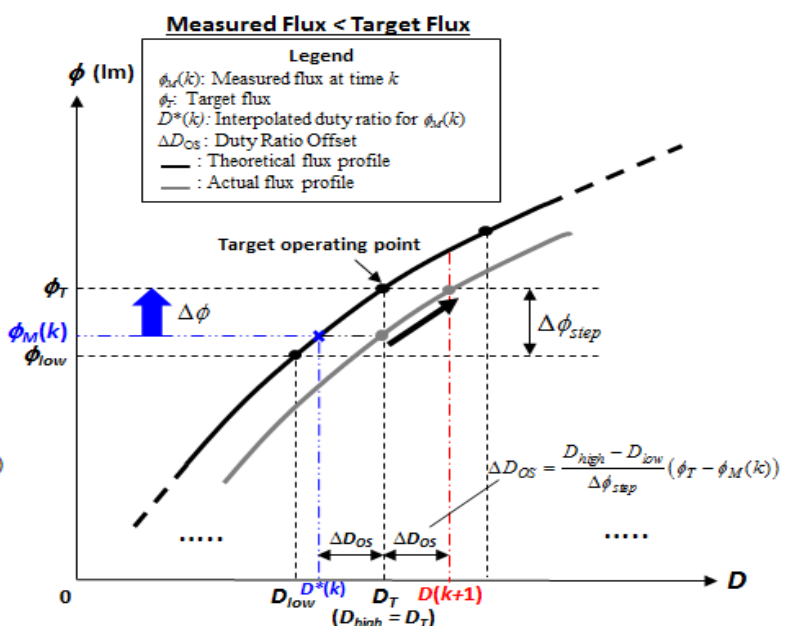

(b)

Fig. 8. Piecewise linear interpolation for determining the new duty ratio when (a) the measured flux is larger than the target flux and (b) the measured flux is smaller than the target flux.

Suppose the measured flux is larger than the target flux. That is, for a given duty ratio of $D_{T}$, the measured flux value $\phi_{\mathrm{M}}(k)$ is greater than the theoretical flux value of $\phi_{T}$ obtained from the nonlinear model. There is an inherent offset between the actual flux profile and the theoretical flux profile as depicted in Fig. 8a. In order to compensate for this offset, the target duty ratio $D_{T}$ needs to be reduced by $\Delta D_{O S}$ in order to bring the actual flux value closer to the target one. A hypothetical duty ratio $D^{*}(k)$ is defined which corresponds to the measured flux $\phi_{\mathrm{M}}(k)$ based on the theoretical flux profile. The duty cycle offset $\Delta D_{O S}$ is defined as $D^{*}(k)-D_{T}$. The point-slope form of the piecewise linear function containing the two points ( $\left.\phi_{h i g h}, D_{h i g h}\right)$ and $\left(\phi_{T}, D_{T}\right)$ is given by

$$
\text { Slope }=\frac{\phi_{\text {high }}-\phi_{T}}{D_{\text {high }}-D_{T}}=\frac{\phi_{M}(k)-\phi_{T}}{D^{*}(k)-D_{T}}=\frac{\phi_{M}(k)-\phi_{T}}{\Delta D_{O S}}
$$

By re-arranging the terms in (11), the duty cycle offset $\Delta D_{o s}$ can be obtained as

$$
\Delta D_{O S}=\frac{D_{\text {high }}-D_{T}}{\phi_{\text {high }}-\phi_{T}}\left[\phi_{M}(k)-\phi_{T}\right]=\frac{D_{\text {high }}-D_{T}}{\Delta \phi_{S T E P}}\left[\phi_{M}(k)-\phi_{T}\right]
$$

where $\Delta \phi_{\text {STEP }}=\phi_{\text {high }}-\phi_{T}=\phi_{T}-\phi_{\text {low }}$. From Fig. 8a, the new duty ratio at the next sampling time instant $D(k+1)$ is expressed as 


$$
D(k+1)=D_{T}-\Delta D_{O S}=D_{T}-\frac{D_{h i g h}-D_{T}}{\Delta \phi_{S T E P}}\left[\phi_{M}(k)-\phi_{T}\right]
$$

Similarly, when the measured flux is smaller than the target flux as depicted in Fig. 8b, the new duty ratio at the next sampling time instant $D(k+1)$ can be written as

$$
D(k+1)=D_{T}+\Delta D_{O S}=D_{T}+\frac{D_{T}-D_{\text {low }}}{\Delta \phi_{\text {STEP }}}\left[\phi_{T}-\phi_{M}(k)\right]
$$

It is important to note that the actual and theoretical flux profiles shown in Fig. 8 are based on the same CCT value. That is, the overall CCT remains unchanged during the flux correction. Under real operating conditions, the actual flux profile of an LED is not fixed. For example, it can be shifted above or below the reference (theoretical) flux profile across the operating lifetime of an LED due to various external factors such as changes in ambient temperature and/or ambient light as well as LED aging. Therefore, the proposed feedback control becomes necessary since it dynamically adjusts the duty ratio in such a way that actual flux value is in close proximity to the target flux value. Since this is an iterative process, $D_{T}$ in (13) and (14) represents only the initial value of duty ratio, i.e. $D_{T}=D(k=0)$. In general, $D_{T}$ should be replaced by the present duty ratio $D(k)$. $D_{\text {high }}$ and $D_{\text {low }}$ should also be replaced by $D_{\text {high }}(k)$ and $D_{\text {low }}(k)$, respectively. Hence, the future duty ratio $D(k+1)$ can be expressed in terms of the current duty ratio $D(k)$ as follows.

$$
D(k+1)= \begin{cases}D(k)-\frac{D_{\text {high }}(k)-D_{T}}{\Delta \phi_{\text {step }}}\left(\phi_{M}(k)-\phi_{T}\right) & \text { for } \phi_{M}(k)>\phi_{T} \\ D(k)+\frac{D_{T}-D_{\text {low }}(k)}{\Delta \phi_{\text {step }}}\left(\phi_{T}-\phi_{M}(k)\right) & \text { for } \phi_{M}(k)<\phi_{T}\end{cases}
$$

where $D_{\text {high }}(k)$ and $D_{\text {low }}(k)$ are the two neighboring duty ratios closest to $D(k)$. $\phi_{M}(k)$ is the measured flux from the color sensor at time $=k$ and $\phi_{T}$ is the target flux. In particular, for flux control of a bi-color white LED lamp, the next duty ratios at time $k+1$ for the cool-white and warm-white LEDs, i.e. $D_{c}(k+1)$ and $D_{w}(k+1)$, can be mathematically written as follows.

$$
D_{c}(k+1)= \begin{cases}D_{c}(k)-\frac{D_{c, \text { high }}(k)-D_{c, T}}{\Delta \phi_{\text {step }}}\left(\phi_{M}(k)-\phi_{T}\right) & \text { for } \phi_{M}(k)>\phi_{T} \\ D_{c}(k)+\frac{D_{c, T}-D_{c, \text { low }}(k)}{\Delta \phi_{\text {step }}}\left(\phi_{T}-\phi_{M}(k)\right) & \text { for } \phi_{M}(k)<\phi_{T}\end{cases}
$$




$$
D_{w}(k+1)= \begin{cases}D_{w}(k)-\frac{D_{w, h i g h}(k)-D_{w, T}}{\Delta \phi_{\text {step }}}\left(\phi_{M}(k)-\phi_{T}\right) & \text { for } \phi_{M}(k)>\phi_{T} \\ D_{w}(k)+\frac{D_{w, T}-D_{w, \text { low }}(k)}{\Delta \phi_{\text {step }}}\left(\phi_{T}-\phi_{M}(k)\right) & \text { for } \phi_{M}(k)<\phi_{T}\end{cases}
$$

where $D_{\mathrm{c}}(k)$ and $D_{w}(k)$ represent the duty ratios for the cool-white and warm-white LEDs at the current sampling cycle. $D_{C, T}$ and $D_{w, T}$ represent the target duty ratios for cool-white and warm-white LEDs whose values are fixed throughout the closed-loop operation. For $\phi_{M}(k)>\phi_{T}(k)$, equations (16a) and (16b) imply that $D_{c}(k+1)<D_{c}(k)$ and $D_{w}(k+1)<D_{w}(k)$. For $\phi_{M}(k)<\phi_{T}(k), D_{c}(k+1)>D_{c}(k)$ and $D_{w}(k+1)>D_{w}(k)$. Since the actual flux profile resembles the theoretical flux profile with a vertical offset, it can be represented mathematically by (1). For the case of $\phi_{M}(k)>\phi_{T}(k)$, it can be shown analytically that $\phi_{M}(k+1)<\phi_{M}(k)$. In other words, the measured flux at the next sampling time instant should be smaller than that at the current time instant because of a reduced duty ratio. The measured flux at time $k$ can be obtained from (1) as follows.

$$
\phi_{M}\left(D_{c}(k), D_{w}(k)\right)=\left(\phi_{c 0}+\phi_{w 0}\right)-\alpha_{c} e^{-\left[\beta_{c} D_{w}(k)+\beta_{c, \min }\right] D_{c}(k)}-\alpha_{w} e^{-\left[\beta_{w} D_{c}(k)+\beta_{w, \min }\right] D_{w}(k)}
$$

Likewise, the measured flux at time $k+1$ can be expressed as

$$
\phi_{M}\left(D_{c}(k+1), D_{w}(k+1)\right)=\left(\phi_{c 0}+\phi_{w 0}\right)-\alpha_{c} e^{-\left[\beta_{c} D_{w}(k+1)+\beta_{c, \min }\right] D_{c}(k+1)}-\alpha_{w} e^{-\left[\beta_{w} D_{c}(k+1)+\beta_{w, \min }\right] D_{w}(k+1)}
$$

By subtracting (18) from (17), we have

$$
\begin{aligned}
& \Delta \phi_{M}=\phi_{M}(k)-\phi_{M}(k+1)= \\
& \alpha_{c}\left(e^{-\left[\beta_{c} D_{c}(k+1) D_{w}(k+1)+\beta_{c, \text { min }} D_{c}(k+1)\right]}-e^{-\left[\beta_{c} D_{c}(k) D_{w}(k)+\beta_{c, \min } D_{c}(k)\right]}\right)+\alpha_{w}\left(e^{-\left[\beta_{w} D_{c}(k+1) D_{w}(k+1)+\beta_{w, \text { min }} D_{w}(k+1)\right]}-e^{-\left[\beta_{w} D_{c}(k) D_{w}(k)+\beta_{w, \min } D_{w}(k)\right]}\right)
\end{aligned}
$$

Since $D_{c}(k+1)<D_{c}(k)$ and $D_{w}(k+1)<D_{w}(k), \Delta \phi_{M}=\phi_{M}(k)-\phi_{M}(k+1)>0$. Hence, $\phi_{M}(k+1)<\phi_{M}(k)$.

Likewise, if the measured flux is smaller than the target flux, i.e. $\phi_{M}(k)<\phi_{T}(k), \Delta \phi_{M}=\phi_{M}(k)-\phi_{M}(k+1)<0$ and therefore, $\phi_{M}(k+1)>\phi_{M}(k)$.

It is interesting to note that for the case of flux control, both $D_{c}$ and $D_{w}$ move in the same direction as the flux value. In other words, an increasing (or decreasing) duty ratio of either the warm-white or cool-white LED will result in an increasing (or decreasing) flux value. However, for the case of CCT control, $D_{c}$ and $D_{w}$ move in the opposite direction with respect to the overall CCT value. For instance, a larger value of $D_{c}$ and a smaller value of $D_{w}$ will increase the CCT value, i.e. it becomes more bluish white. Similarly, a smaller value of $D_{c}$ 
and a larger value of $D_{w}$ will reduce the CCT value, i.e. it becomes more yellowish white. The relationship between the combined CCT value and the two duty ratios $\left(D_{c}, D_{w}\right)$ is graphically illustrated in Fig. 7a and $7 \mathrm{~b}$. Based on piecewise linear interpolation, the new duty ratio at the next sampling time instant for the cool-white (or warm-white LED) can be analytically derived in a similar manner as that for the flux value.

Consider the first scenario where the measured CCT value is larger than the target CCT value (i.e. $C C T_{M}>$ $C C T_{T}$ ). Fig. 9 depicts the interpolated duty ratio for (a) cool-white and (b) warm-white LED when the measured CCT is larger than the target CCT for a particular flux value. The hypothetical duty ratios, i.e. $D_{c}{ }^{*}(k)$ and $D_{w} *(k)$, corresponding to the measured CCT, are obtained by doing piecewise linear interpolation between the target operating point and the neighboring operating point along the theoretical CCT profile. For coolwhite LED, the neighboring operating point is chosen to be $\left(D_{c, h i g h}, C C T_{h i g h}\right)$ whereas for warm-white LED, the neighboring operating point is chosen to be $\left(D_{w, l o w}, C C T_{\text {high }}\right)$, where $D_{c, \text { high }}>D_{c, T}, D_{w, l o w}<D_{w, T}$ and $C C T_{\text {high }}=$ $C C T_{T}+\triangle C C T_{S T E P}$. The duty ratio offset $\Delta D_{O S}$ can therefore be readily determined, i.e. $\Delta D_{O S}=D_{c} *(k)-D_{c, T}$ for cool-white LED and $\Delta D_{O S}=D_{w, T}-D_{w} *(k)$ for warm-white LED. This inherent offset accounts for the difference between the actual CCT profile and the theoretical one which must be compensated in order to achieve good agreement between the measured and target CCT values.

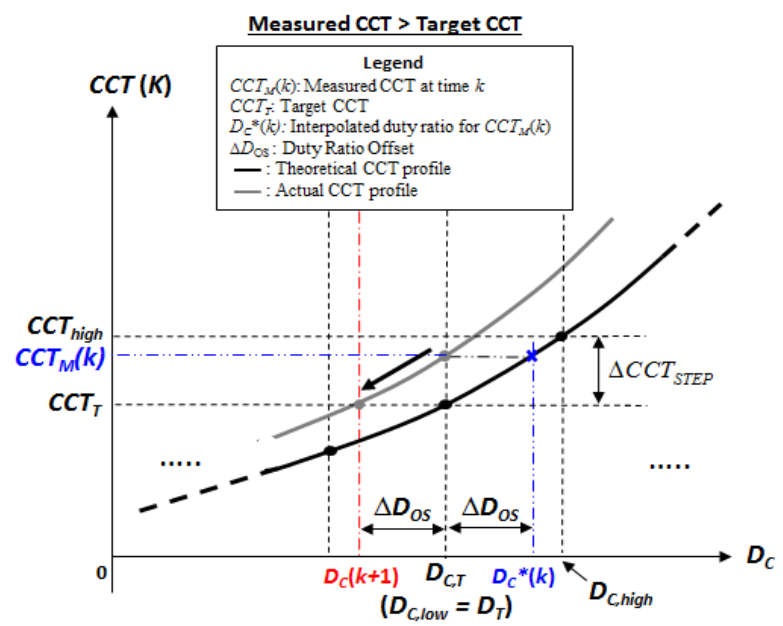

(a)

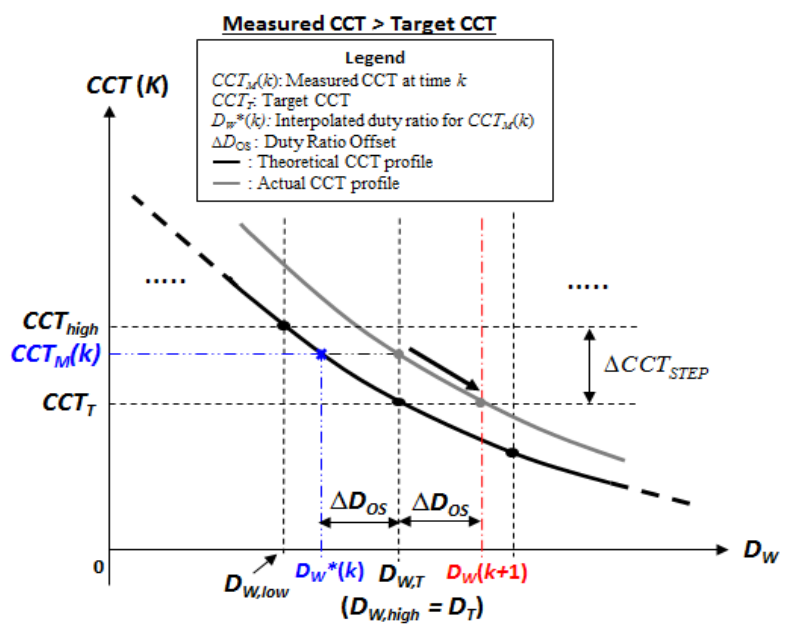

(b)

Fig. 9. Piecewise linear interpolation for determining the new duty ratio of (a) cool-white LED and (b) warm-white LED when the measured CCT is larger than the target CCT. 
Note that the actual and theoretical CCT profiles shown in Fig. 9 assume a constant flux value. In other words, the flux value remains largely unaffected by any change in the CCT value. Consider the opposite case where the measured CCT is smaller than the target CCT (i.e. $C C T_{M}<C C T_{T}$ ). Fig. 10 shows the interpolated the duty ratio for (a) cool-white and (b) warm-white LED.

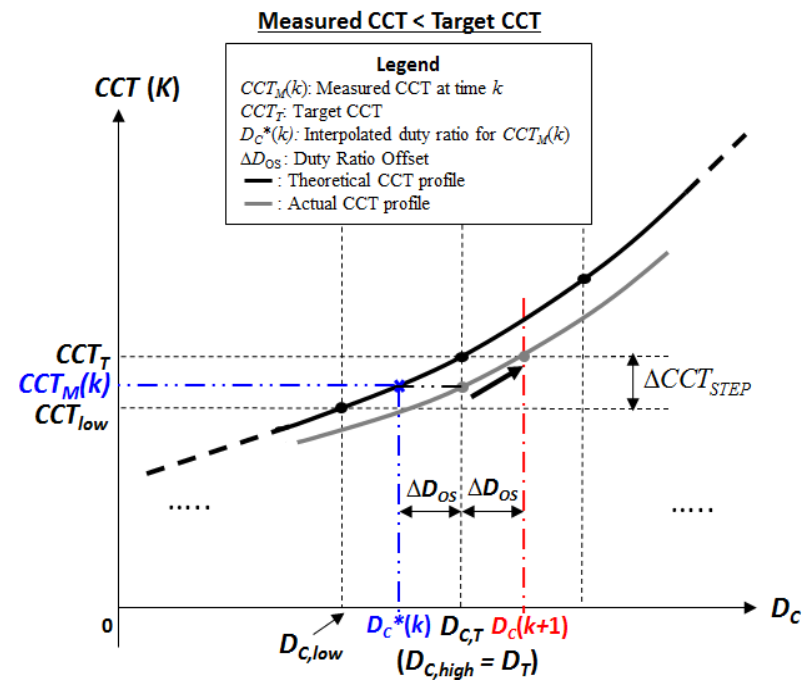

(a)

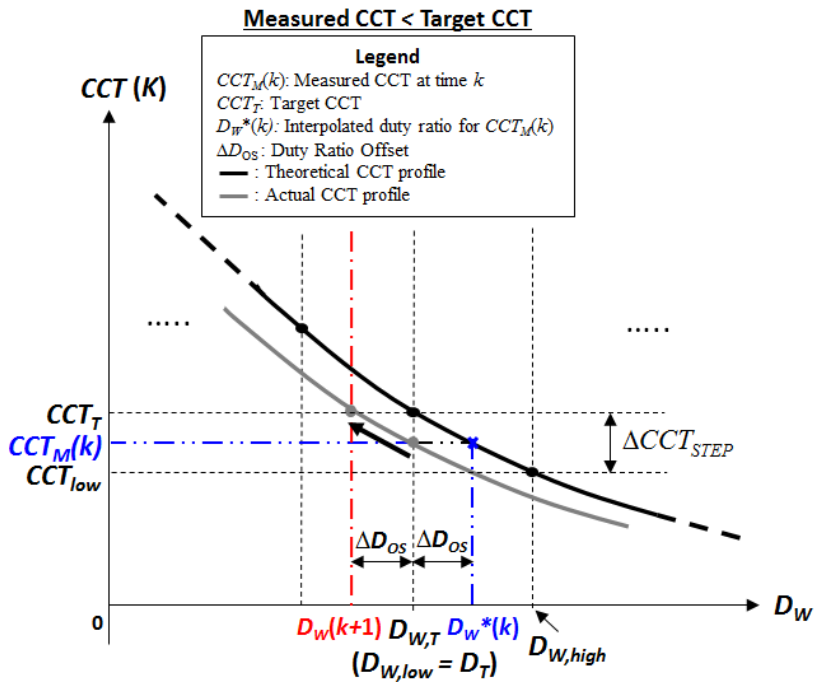

(b)

Fig. 10. Piecewise linear interpolation for determining the new duty ratio of (a) cool-white LED and (b) warm-white LED when the measured CCT is smaller than the target CCT.

Mathematically, the interpolated duty ratios at time $k+1$ for the cool-white and warm-white LEDs, i.e. $D_{c}(k+1)$ and $D_{w}(k+1)$, can be represented as

$$
\begin{gathered}
D_{c}(k+1)= \begin{cases}D_{c}(k)-\frac{D_{c, \text { high }}(k)-D_{c, T}}{\Delta C C T_{\text {step }}}\left(C C T_{M}(k)-C C T_{T}\right) & \text { for } C C T_{M}(k)>C C T_{T} \\
D_{c}(k)+\frac{D_{c, T}-D_{c, \text { low }}(k)}{\Delta C C T_{\text {sep }}}\left(C C T_{M}(k)-C C T_{T}\right) & \text { for } C C T_{M}(k)<C C T_{T}\end{cases} \\
D_{w}(k+1)= \begin{cases}D_{w}(k)+\frac{D_{w, T}-D_{w, \text { low }}(k)}{\Delta C C T_{\text {sep }}}\left(C C T_{M}(k)-C C T_{T}\right) & \text { for } C C T_{M}(k)>C C T_{T} \\
D_{w}(k)-\frac{D_{w, h i g h}-D_{w, T}}{\Delta C C T_{\text {step }}}\left(C C T_{M}(k)-C C T_{T}\right) & \text { for } C C T_{M}(k)<C C T_{T}\end{cases}
\end{gathered}
$$

The ratio of $D_{c}$ and $D_{w}$ can be adjusted in order to vary the overall color temperature of the bi-color lamp at a particular flux value. It has been shown in [19] that at a particular total duty ratio $D_{T}\left(0 \leq D_{T}=D_{c}+D_{w} \leq 2\right)$, the CCT for the cool-white LED, denoted by $C C T_{c}$, becomes smaller with a smaller value of $D_{c}$ and a larger 
value of $D_{w}$ and vice versa. However, the CCT for the warm-white LED, denoted by $C C T_{w}$, is largely independent on the ratio of $D_{c}$ and $D_{w}$ at any particular value of $D_{T}$. It can simply be modeled as a function of $D_{T}$ using a straight line, i.e. $C C T_{w}$ is assumed to be constant at a fixed value of $D_{T}$. In theory, for constant flux, $D_{T}$ should remain unchanged during the adjustment of the overall CCT. In other words, $\phi_{c}, \phi_{w}$ and $C C T_{w}$ are assumed to be constant $\left(C C T_{w}=C C T_{W 0}\right)$. Hence, Equation (10) can be re-written as

$$
\operatorname{CCT}_{M}\left(D_{c}, D_{w}\right)=\frac{\phi_{C 0}+\phi_{W 0}}{\frac{\phi_{C 0}}{C C T_{C}\left(D_{c}, D_{w}\right)}+\frac{\phi_{W 0}}{C C T_{W 0}}} \quad \text { for } D_{T}=D_{T 0}
$$

where $C C T_{c}=k_{1} D_{c}-k_{2} D_{w}=k_{1} D_{c}-k_{2}\left(D_{T 0}-D_{c}\right)=\left(k_{1}+k_{2}\right) D_{c}-k_{2} D_{T 0}$ and $k_{1}$ and $k_{2}$ are constant.

As shown in Fig. 9, if the measured CCT is larger than the target CCT (i.e. $C C T_{M}(k)>C C T_{T}$ ), the duty ratio of the cool-white LED is reduced, i.e. $D_{c}(k+1)<\mathrm{D}_{c}(k)$ whereas that of the warm-white LED is increased, i.e. $D_{w}(k+1)>D_{w}(k)$. This will effectively reduce the value of $C C T_{C}\left(D_{c}, D_{w}\right)$. As a result, the combined CCT will become smaller as given by (21). This will bring the measured CCT at the next sampling time instant closer to the target CCT. Similarly, if the measured CCT is smaller than the target CCT (i.e. $C C T_{M}(k)<C C T_{T}$ ), the duty ratio of the cool-white LED is increased, i.e. $D_{c}(k+1)>D_{c}(k)$ whereas that of the warm-white LED is reduced, i.e. $D_{w}(k+1)<D_{w}(k)$. This will effectively increase the value of $C C T_{C}\left(D_{c}, D_{w}\right)$. Consequently, the combined CCT will increase as given by (21). Hence, the measured CCT will eventually converge to the target CCT in steady-state condition. For the special case in which the measured flux or CCT is identical to the target flux or CCT, no interpolation method is necessary.

In existing linear control approaches, the control of flux and CCT values are governed by the following equations.

$$
\left\{\begin{array}{l}
\phi_{M}=\phi_{W}+\phi_{C} \\
C C T_{M}=C C T_{W} \frac{\phi_{W}}{\phi_{W}+\phi_{C}}+C C T_{C} \frac{\phi_{C}}{\phi_{W}+\phi_{C}}
\end{array}\right.
$$

where $\phi_{M}$ and $C C T_{M}$ are the measured values of the overall flux and CCT, respectively. $\phi_{W}$ and $C C T_{W}$ are the average luminous flux and CCT values associated with the warm-white LED. $\phi_{C}$ and $C C T_{C}$ are the average 
luminous flux and CCT values corresponding to the cool-white LED. By re-arranging the terms in (22), $\phi_{W}$ and $\phi_{C}$ can be expressed in terms of $\phi_{M}, C C T_{M}, C C T_{W}$ and $C C T_{C}$ as follows.

$$
\left\{\begin{array}{l}
\phi_{C}=\frac{\phi_{M}\left(C C T_{M}-C C T_{W}\right)}{C C T_{C}-C C T_{W}} \\
\phi_{W}=\frac{\phi_{M}\left(C C T_{C}-C C T_{M}\right)}{C C T_{C}-C C T_{W}}
\end{array}\right.
$$

Also, the duty ratios at the next sampling time instant, i.e. $D_{c}(k+1)$ and $D_{w}(k+1)$, can be expressed as

$$
\left\{\begin{array}{l}
D_{c}(k+1)=D_{c}(k)+\Delta D_{c}=D_{c}(k)+\frac{1}{\eta_{c}} \Delta \phi_{c} \\
D_{w}(k+1)=D_{w}(k)+\Delta D_{w}=D_{w}(k)+\frac{1}{\eta_{w}} \Delta \phi_{w}
\end{array}\right.
$$

where $D_{c}(k)$ and $D_{\mathrm{w}}(k)$ are the duty ratios at the current sampling time instant. $\Delta D_{c}$ and $\Delta D_{w}$ are the change in the duty ratios. $\Delta \phi_{c}$ and $\Delta \phi_{w}$ are the respective change in the average luminous flux for cool-white and warmwhite LEDs. $\eta_{c}$ and $\eta_{w}$ represent the proportionality constant between the duty ratio and the luminous flux which can be estimated by "curve-fitting" the experimental data using a straight line. Now, the key is to determine $\Delta \phi_{c}$ and $\Delta \phi_{w}$ which enables us to calculate the necessary change in the duty ratio for the next sampling time instant. By using equation (23), $\Delta \phi_{c}$ and $\Delta \phi_{w}$ can be represented as

$$
\left\{\begin{array}{l}
\Delta \phi_{C}=\frac{\left(\phi_{T}-\phi_{M}\right)\left(C C T_{M}-C C T_{W}\right)}{C C T_{C}-C C T_{W}}=\frac{\Delta \phi\left(C C T_{M}-C C T_{W}\right)}{C C T_{C}-C C T_{W}} \\
\Delta \phi_{W}=\frac{\left(\phi_{T}-\phi_{M}\right)\left(C C T_{C}-C C T_{M}\right)}{C C T_{C}-C C T_{W}}=\frac{\Delta \phi\left(C C T_{C}-C C T_{M}\right)}{C C T_{C}-C C T_{W}}
\end{array}\right.
$$

where $\Delta \phi$ denotes the difference between the target flux and the measured flux. If the measured flux is larger than the target flux, both $\Delta \phi_{c}$ and $\Delta \phi_{w}$ become negative which means that the duty ratios for both cool-white and warm-white LEDs have to be reduced for the next sampling time instant. On the other hand, if the measured flux is smaller than the target flux, both $\Delta \phi_{c}$ and $\Delta \phi_{w}$ become positive which means that the duty ratios need to be increased for the next sampling time instant. 
Likewise, $\Delta \phi_{c}$ and $\Delta \phi_{w}$ due to the difference between the measured CCT and target CCT can be written as

$$
\left\{\begin{array}{l}
\Delta \phi_{C}=\frac{\phi_{M}\left(C C T_{T}-C C T_{M}\right)}{C C T_{C}-C C T_{W}} \\
\Delta \phi_{W}=\frac{\phi_{M}\left(C C T_{M}-C C T_{T}\right)}{C C T_{C}-C C T_{W}}
\end{array}\right.
$$

Suppose the measured CCT is larger than the target CCT. $\Delta \phi_{c}$ is negative which implies that the duty ratio for the cool-white LED is reduced. In contrast, $\Delta \phi_{w}$ is positive which implies that the duty ratio for the warm-white LED is increased. By combining (25) and (26), the total change in the average luminous flux in terms of both the flux and CCT errors can be represented as

$$
\left\{\begin{array}{l}
\Delta \phi_{C, t o t}=\frac{\phi_{M}\left(C C T_{T}-C C T_{W}\right)+\phi_{T}\left(C C T_{W}-C C T_{M}\right)}{C C T_{C}-C C T_{W}} \\
\Delta \phi_{W, t o t}=\frac{\phi_{M}\left(C C T_{T}-C C T_{C}\right)+\phi_{T}\left(C C T_{M}-C C T_{C}\right)}{C C T_{C}-C C T_{W}}
\end{array}\right.
$$

Hence, from (24) and (27), the general form of the duty ratios at the next sampling time instant are given by

$$
\left\{\begin{array}{l}
D_{c}(k+1)=D_{c}(k)+\frac{1}{\eta_{c}}\left[\frac{\phi_{M}\left(C C T_{T}-C C T_{W}\right)+\phi_{T}\left(C C T_{W}-C C T_{M}\right)}{C C T_{C}-C C T_{W}}\right] \\
D_{w}(k+1)=D_{w}(k)+\frac{1}{\eta_{w}}\left[\frac{\phi_{M}\left(C C T_{T}-C C T_{C}\right)+\phi_{T}\left(C C T_{M}-C C T_{C}\right)}{C C T_{C}-C C T_{W}}\right]
\end{array}\right.
$$

Basically, the control law for regulating the flux and CCT using the closed-loop linear approach is governed by equation (28).

\section{IMPLEMENTATION OF THE BI-COLOR ADJUSTABLE LED SYSTEM}

The key building blocks of the bi-color adjustable LED lamp are discussed in this section. The MCU is a critical component in the feedback loop as it continuously generates the PWM duty ratios for driving the coolwhite and warm-white LED strings. A high-performance, low-power and low-cost Atmel AVR 8-bit microcontroller (part number: Atmega32A) is used. This particular MCU runs on a $1 \mathrm{MHz}$ internal oscillator 
which is fast enough for general applications. The control algorithm for performing the closed-loop nonlinear control is implemented using the $\mathrm{C}$ language. Fig. 11 contains a simplified flowchart for illustrating the underlying algorithm of the proposed nonlinear controller for the bi-color white LED lamp.

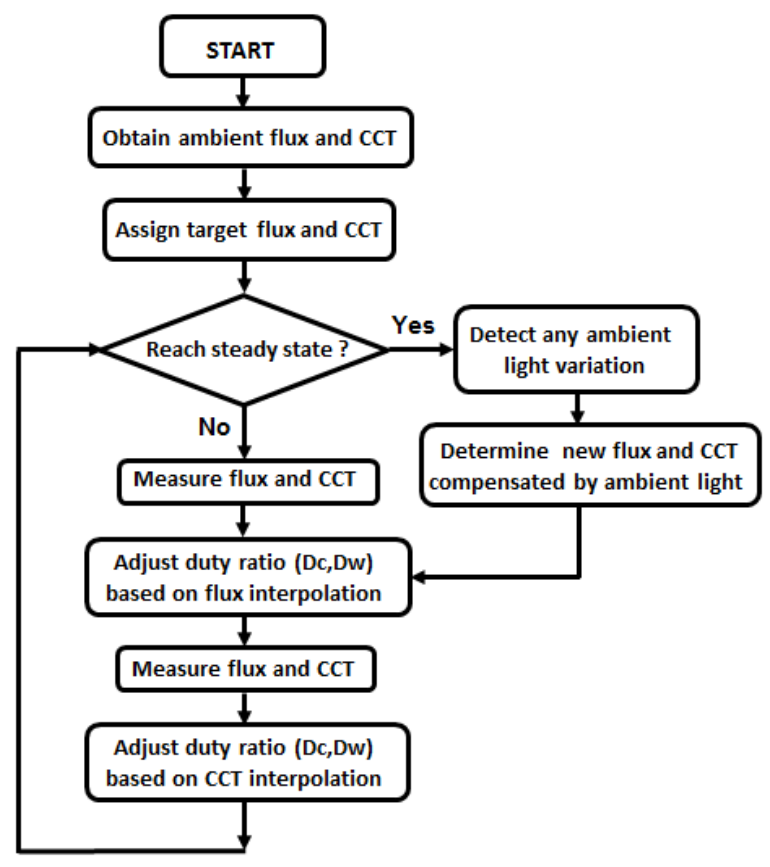

Fig. 11. Simplified flowchart for illustrating the algorithm of the proposed nonlinear controller.

During start-up, the RGB values associated with the ambient light are generated by the color sensor with the LEDs completely switched OFF. Based on the target values for flux and CCT specified by the user, the LED system is then initialized and switched ON with the calibrated duty ratios for the cool-white and warmwhite LEDs. Under normal operation, the embedded color sensor will continuously monitor the actual flux and CCT. Assuming a constant CCT, the MCU will perform a first round of piecewise linear interpolation based on the difference between the actual and target flux values in order to determine a new set of duty ratios $\left(D_{c}\right.$, $D_{w}$ ) for regulating the luminous flux. After fixing the flux value, the MCU will perform a second round of piecewise linear interpolation based on the difference between the actual and target CCT values in order to obtain a new set of duty ratios $\left(D_{c}, D_{w}\right)$ for regulating the CCT. This tuning process is repeated over time. The closed-loop system will ultimately reach steady-state condition after a few iterations in which the actual flux and CCT values are in close proximity with the corresponding target values. To account for any variations in the ambient light, an additional RGB measurement is performed on a regular basis so that the inherent RGB 
offset can be extracted for ambient light compensation. Suppose the initial RGB values associated with the ambient light are denoted by $\left(R_{0}, G_{0}, B_{0}\right)$. Hence, the net RGB values, i.e. $\left(R_{L E D}(k), G_{L E D}(k), B_{L E D}(k)\right)$, contributed by the LED lamp at time $k$ are given by

$$
\begin{aligned}
& R_{L E D}(k)=R_{m}(k)-R_{0} \\
& G_{L E D}(k)=G_{m}(k)-G_{0} \\
& B_{L E D}(k)=B_{m}(k)-B_{0}
\end{aligned}
$$

where $\left(R_{m}(k), G_{m}(k), B_{m}(k)\right)$ are the measured RGB values from the color sensor at time $k$. Fig. 12 shows the response spectra of the $\mathrm{R}, \mathrm{G}$, and B sensors [27].

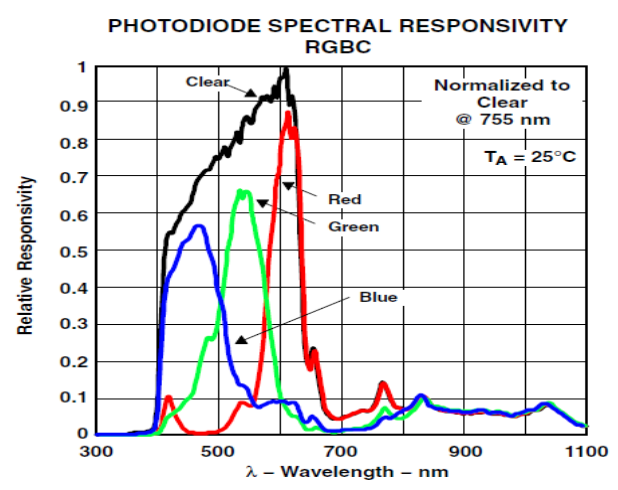

Fig. 12. Spectral responsivity of red (R), green (G) and blue (B) sensors [27].

Equation (29) represents the static compensation of RGB values for the case of constant ambient light condition. Suppose the RGB values associated with the ambient light is changed to $\left(R_{l}, G_{l}, B_{l}\right)$ at time $k+1$, where $R_{l}=R_{0}+\Delta R_{a}, G_{l}=G_{0}+\Delta G_{a}$, and $B_{l}=B_{0}+\Delta B_{a}$. Since the RGB values from the LED lamp remain unchanged, any difference between the measured values of $\left(R_{m}(k+1), G_{m}(k+1), B_{m}(k+1)\right)$ and $\left(R_{m}(k), G_{m}(k)\right.$, $\left.B_{m}(k)\right)$ is caused by the variation in the background light. Mathematically, it can be expressed as

$$
\begin{aligned}
& \Delta R_{a}=R_{m}(k+1)-R_{m}(k) \\
& \Delta G_{a}=G_{m}(k+1)-G_{m}(k) \\
& \Delta B_{a}=B_{m}(k+1)-B_{m}(k)
\end{aligned}
$$

where $\Delta R_{a}, \Delta G_{a}$ and $\Delta B_{a}$ represent the change in the RGB values of the ambient light. Therefore, the effective RGB values required for the LED lamp at time $k+1$ can be written as 


$$
\begin{aligned}
& R_{L E D}(k+1)=R_{m}(k+1)-R_{1}=R_{m}(k+1)-\left(R_{0}+\Delta R_{a}\right) \\
& G_{L E D}(k+1)=G_{m}(k+1)-G_{1}=G_{m}(k+1)-\left(G_{0}+\Delta G_{a}\right) \\
& B_{L E D}(k+1)=B_{m}(k+1)-B_{1}=B_{m}(k+1)-\left(B_{0}+\Delta G_{a}\right)
\end{aligned}
$$

Equation (31) represents the dynamic compensation of RGB values for the case of changing ambient light condition. The RGB values obtained from the color sensor are mapped to the CIE tri-stimulus values (XYZ) [20], [24], [28]. The tri-stimulus Y value represents the illuminance (flux). The chromaticity coordinates $(x, y)$ can be calculated from the tri-stimulus values, where $x=\mathrm{X} /(\mathrm{X}+\mathrm{Y}+\mathrm{Z})$ and $y=\mathrm{Y} /(\mathrm{X}+\mathrm{Y}+\mathrm{Z})$. Finally, McCamy's formula can be used to determine the CCT from the chromaticity coordinates. An asynchronous interrupt mechanism is also included in the software which allows the users to perform real-time updates of the desired flux and/or CCT values during the normal operation of the bi-color LED luminaire.

The concept of the proposed closed-loop system is highly dependent upon the selection of the color sensor. The TCS3472 light-to-digital color converter is used which provides a digital return of red, green, blue (RGB) and clear light sensing values [19]. TCS3472 is known for its smallest number of photodiode, i.e., a 3 x 4 photodiode array, compared with a 4 x 4 photodiode array in other widely-used color sensors. In addition, it contains an IR blocking filter which minimizes the IR spectral component of the incoming light, thereby enabling more accurate measurement of flux and color. Communication with the MCU is accomplished easily via the industry standard two-wire $\mathrm{I}^{2} \mathrm{C}$ serial bus. The light bulb comprising the embedded color sensor and LEDs has to be calibrated once using the Integrative Sphere before actual usage. Another important design consideration is the LED die arrangement and sensor location. The LED dies need to be arranged in such a way that the light emitted from each of them can be in a symmetrical fashion. This is quite significant as it will give more accurate readings during calibration with the Integrative Sphere. 


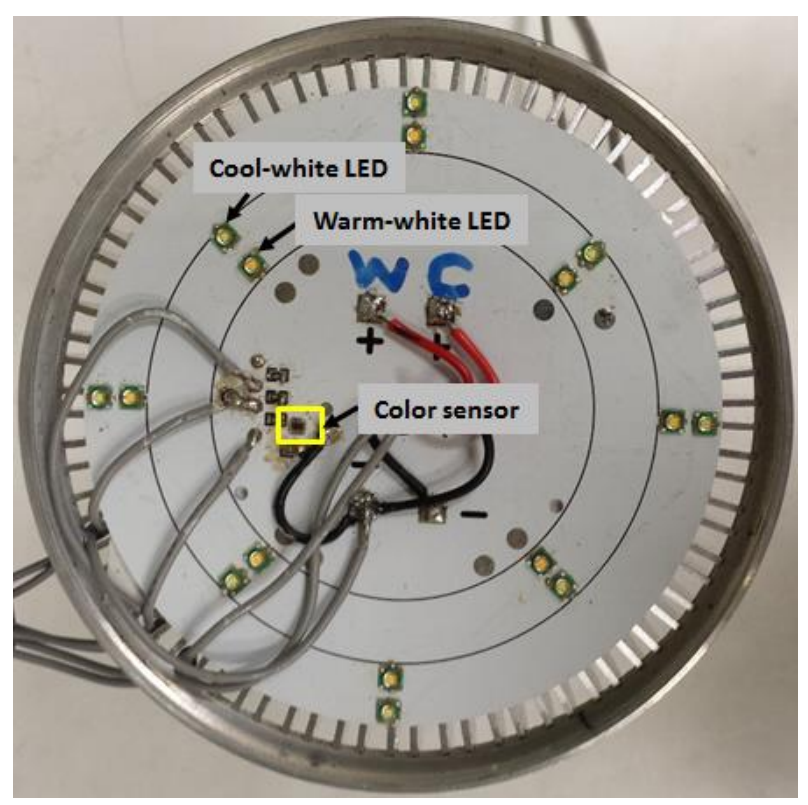

Fig. 13. Photo of the LED PCB showing a symmetrical placement of LED dies to yield good illumination.

Fig. 13 shows the photo of the symmetrical placement of the LED dies to produce good illumination. The layout of the two-string LEDs is largely made up of two circular lanes. The inner lane consists of a total of 8 warm-white LEDs with a CCT ranging from $2600 \mathrm{~K}$ to $3700 \mathrm{~K}$ and the outer lane consists of a total of 8 coolwhite LEDs with a CCT ranging from $5000 \mathrm{~K}$ to $10000 \mathrm{~K}$. Table 5 contains the product specifications of the cool-white and warm-white LEDs from Cree's XLamp XP-E series [29]. Fig. 14 shows the relative spectral power distribution for the cool-white and warm-white LEDs [29]. As shown in Fig. 13, the color sensor is located near the center of the circular PCB and on the same horizontal plane as the LED dies. Since the total area of the LED PCB is relatively small (its diameter is only $9 \mathrm{~cm}$ ), it will receive almost the same amount of light from every LED as long as it is located in close proximity to the center of the PCB. 


\begin{tabular}{|c|c|c|}
\hline Color & Cool-white LED & Warm-white LED \\
\hline \multicolumn{3}{|c|}{ Luminous Flux (lm) } \\
\hline Maximum Flux @ 1 A & 225 & 181.35 \\
\hline Nominal Flux @ 0.35 A & 100 & 80.60 \\
\hline Minimum Flux @ 0.05 A & 12.50 & 10.00 \\
\hline Correlated Color Temperature (CCT) (K) \\
\hline Maximum CCT & 10,000 & 3,700 \\
\hline Minimum CCT & 5,000 & 2,600 \\
\hline \multicolumn{2}{|c|}{0.35} \\
\hline Laximum current & 0.05 \\
\hline Nominal current & 3.5 \\
\hline Minimum current & 1.1 \\
\hline \multicolumn{2}{|c|}{0.1375} \\
\hline Maximum power & Power consumption (W) \\
\hline Nominal power & . \\
\hline Minimum power &
\end{tabular}

Table 5. Product specifications of the cool-white and warm-white LEDs from Cree's XLamp XP-E series [29].

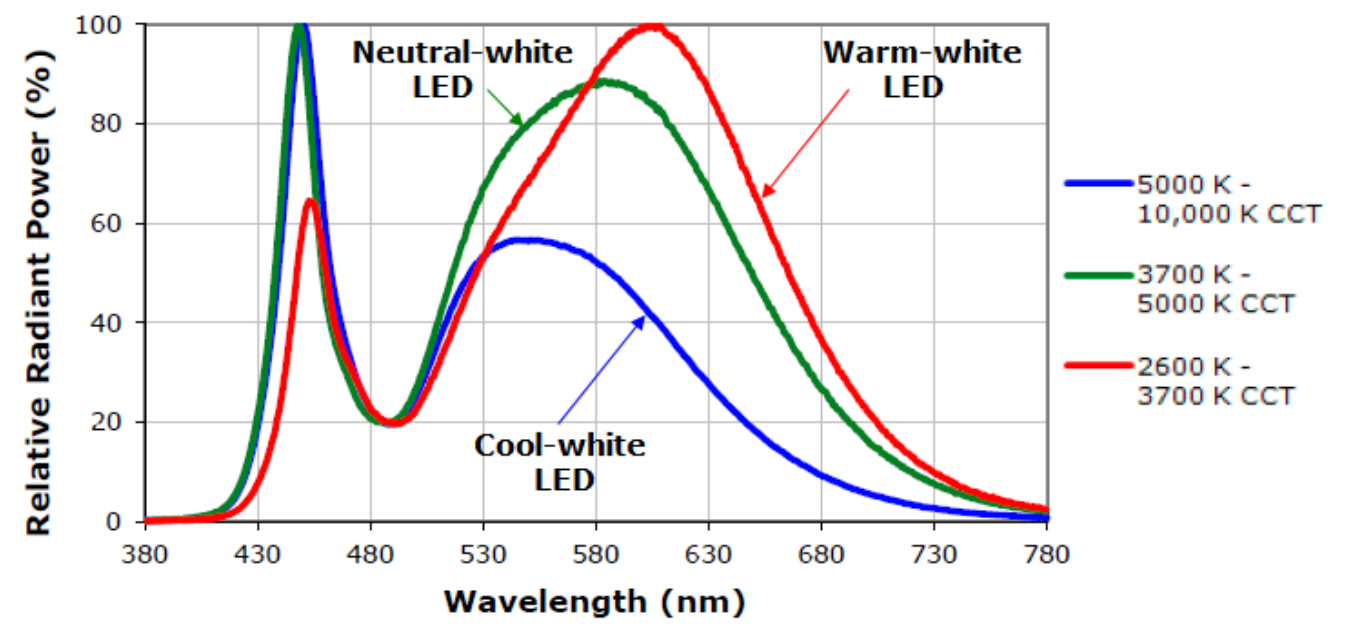

Fig. 14. Relative spectral power distribution of the cool-white, warm-white and neutral-white LEDs [29].

The power stage of this bi-color LED lamp is implemented using one AC-DC converter [30] and two LED drivers [31]. The input voltage range of the AC-DC converter is 85-264 VAC (input frequency is between $47-63 \mathrm{~Hz}$ ). The output voltage is a DC voltage of $48 \mathrm{~V}$ with a nominal current of $0.21 \mathrm{~A}$. The output of the AC-DC converter is connected to a pair of LED drivers, one of which drives the warm-white LED string and the other drives the cool-white LED string. The LED driver is essentially a buck switching regulator which delivers a maximum average current of $500 \mathrm{~mA}$ to high-power LEDs. The MCU supplies a logic-level PWM 
signals to the LED driver for enabling PWM dimming. The dimming ratio ranges from $0.39 \%$ to $100 \%$ which is sufficiently wide for general dimming and color-mixing applications.

\section{EXPERIMENTAL RESULTS}

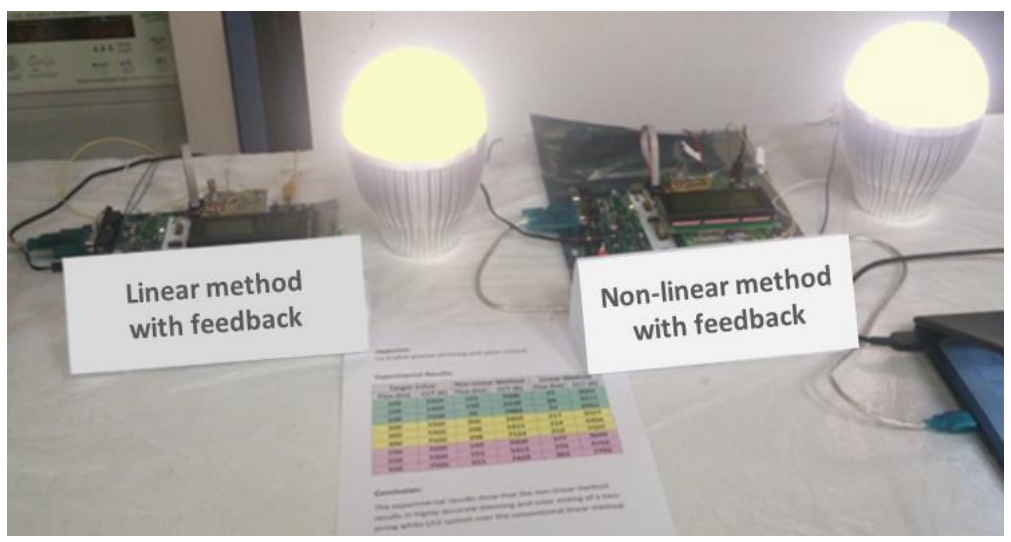

Fig. 15. Experimental setup of the bi-color LED lamp using the closed-loop nonlinear control and the closed-loop linear control scheme.

A photo of the experimental setup is shown in Fig. 15. Both LED lamps shown in Fig. 15 are made up of the same hardware. The only difference between them is the software program in the MCU which defines the underlying control method. The LED lamp on the left uses the conventional linear method with feedback control whereas the LED lamp on the right uses the proposed nonlinear method with feedback control.

The flux and CCT values in steady-state condition of the bi-color LED lamp using the proposed closedloop nonlinear control scheme and its closed-loop linear counterpart are measured at room temperature. Fig. 16 gives a comparison on the measured flux and CCT values versus the corresponding target values between the two control schemes. The experimental results show that the maximum error between the target value and the measured value for flux and CCT based on the proposed nonlinear approach is $1 \%$ and $1.148 \%$, respectively. Basically, the measured values are in good agreement with the corresponding target values. In comparison, the maximum error between the target value and the measured value for flux and CCT based on the linear approach is $15.64 \%$ and $27.46 \%$. Therefore, the proposed closed-loop nonlinear control approach enables very precise dimming and color control of a two-string LED system. In addition to room temperature, the steady-state flux and CCT values of the bi-color LED lamp are also measured at the two extreme 
temperatures, namely $-10^{\circ} \mathrm{C}$ and $55^{\circ} \mathrm{C}$.

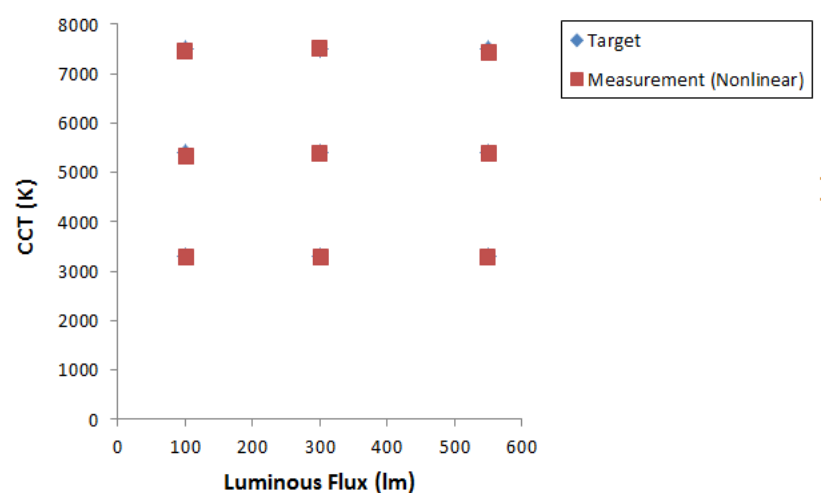

(a)

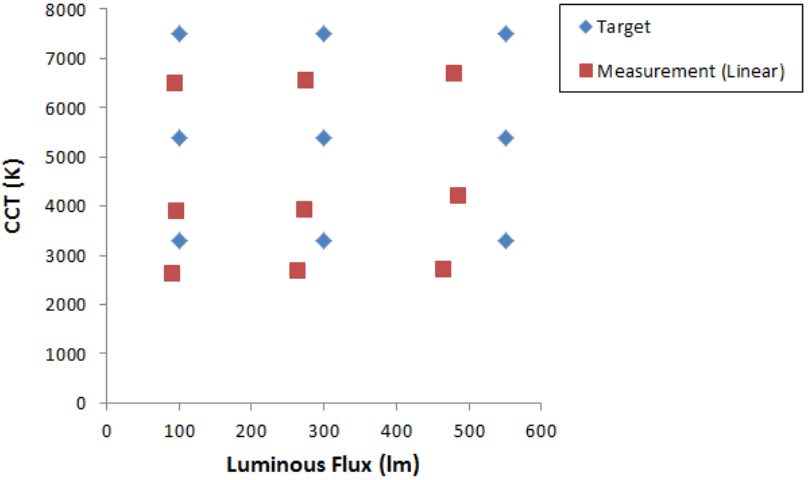

(b)

Fig. 16. Measured and target values of luminous flux and CCT of the bi-color LED lamp at $25^{\circ} \mathrm{C}$ using (a) the proposed closed-loop nonlinear approach and (b) the closed-loop linear approach.

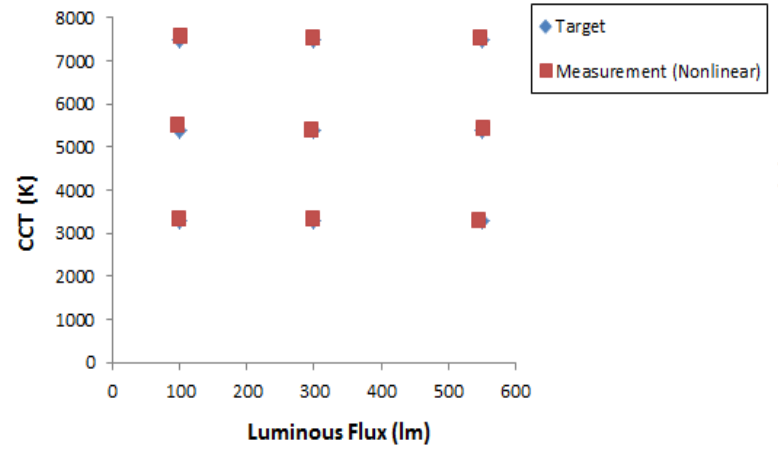

(a)

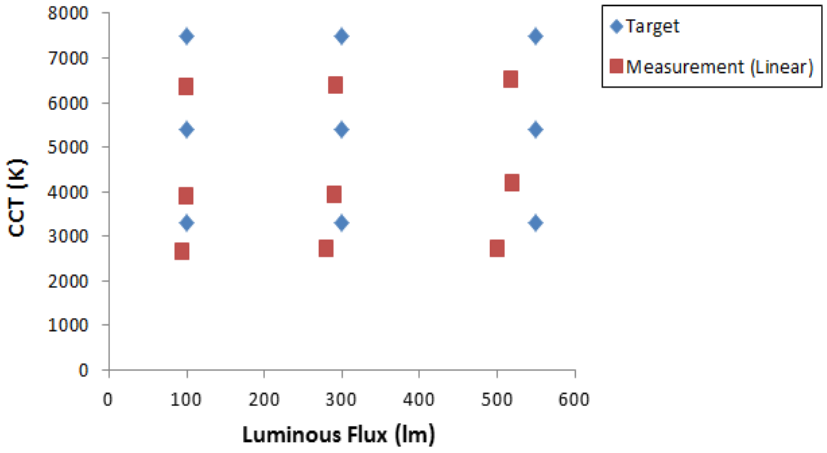

(b)

Fig. 17. Measured and target values of luminous flux and CCT of the bi-color white LED lamp at $-10^{\circ} \mathrm{C}$ using (a) the proposed closed-loop nonlinear approach and (b) the closed-loop linear approach.

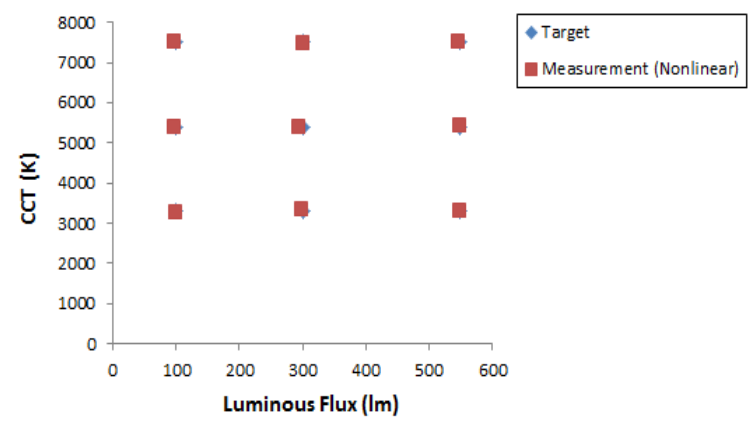

(a)

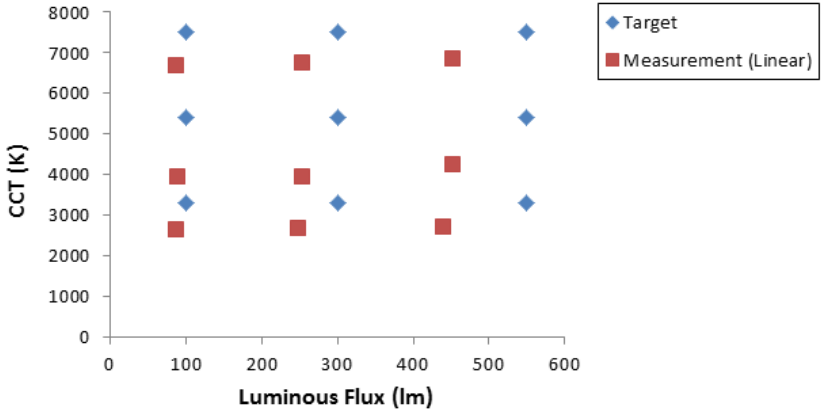

(b)

Fig. 18. Measured and target values of luminous flux and CCT of the bi-color white LED lamp at $55^{\circ} \mathrm{C}$ using (a) the proposed closed-loop nonlinear approach and (b) the closed-loop linear approach. 
Fig. 17 and 18 give a comparison of the measured values and target values of flux and CCT values of the lamp at the respective ambient temperature of $-10^{\circ} \mathrm{C}$ and $55{ }^{\circ} \mathrm{C}$. From the experimental results, it is demonstrated that the bi-color LED lamp with the proposed closed-loop nonlinear control is robust to ambient temperature change, unlike its linear counterpart. The maximum error between the measured and target values of flux and CCT using the closed-loop nonlinear control scheme is $3 \%$ and $1.78 \%$, respectively. In comparison, the maximum error between the measured and target values of flux and CCT using the closedloop linear control scheme is $20 \%$ and $27.5 \%$, respectively. It is also worth noting that the proposed nonlinear control scheme does not require the use of temperature sensor. Yet, it can achieve very precise flux and CCT control across different ambient temperatures.

Besides ambient temperature change, it is also beneficial to investigate the effect of ambient light interferences on the proposed bi-color LED system, especially for outdoor lighting applications. Initially, the ambient light intensity is assumed to be fixed at either $15 \mathrm{~lm}$ or $170 \mathrm{~lm}$. The former case models a weak ambient lighting condition (dark setting) whereas the latter models a strong ambient light condition (bright setting). Fig. 19 shows the measured and target flux and CCT values from the bi-color LED lamp with the proposed closed-loop nonlinear control method in a bright ambient light condition and a dark ambient light condition.

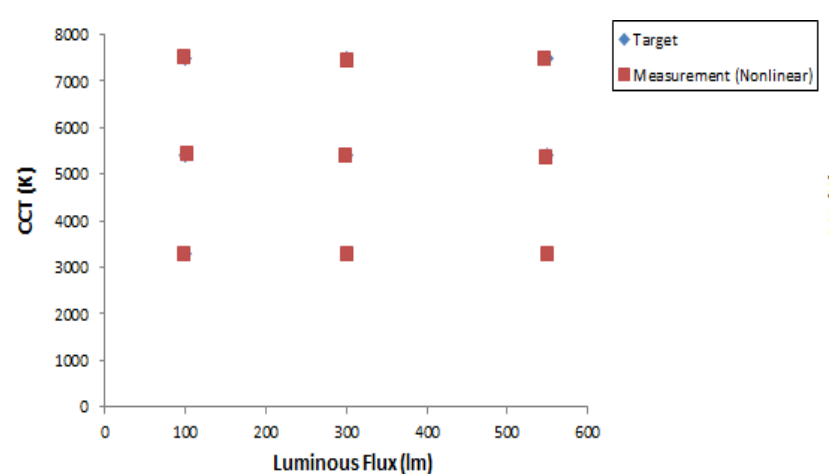

(a)

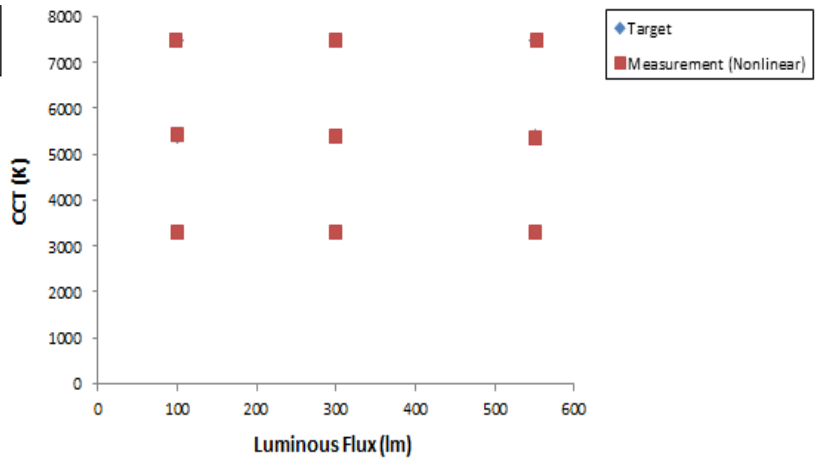

(b)

Fig. 19. Measured and target values of flux and CCT of the bi-color white LED lamp with nonlinear control under (a) bright ambient condition $(170 \mathrm{~lm})$ and (b) dark ambient condition $(15 \mathrm{~lm})$.

The experimental results show that the actual flux and CCT values continue to track closely with the target 
values, independent of the current ambient light intensity level. Also, no ambient light sensor is needed which helps reduce the total cost and power consumption. This confirms the robustness of the proposed ambient light compensation scheme. The maximum error between the target value and the measured value for flux and CCT based on the proposed nonlinear approach is $1 \%$ and $1.148 \%$, respectively.

It is also crucial to study the dynamic behavior of the bi-color LED lamp in response to any changes in the ambient light. Two opposite scenarios are considered, namely the ambient light intensity increases from $7 \mathrm{~lm}$ to $170 \mathrm{~lm}$ and the ambient light intensity decreases from $170 \mathrm{~lm}$ to $7 \mathrm{~lm}$. Fig. 20 shows the measured values of flux and CCT (before and after the transient in ambient light) relative to the target flux in either case.

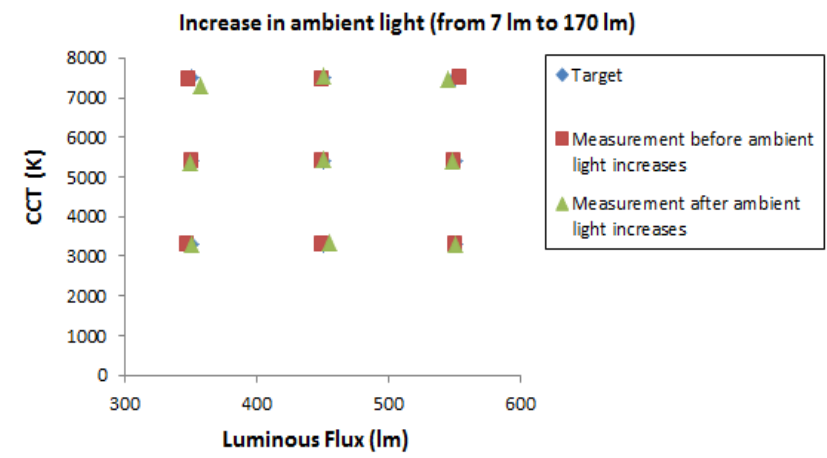

(a)

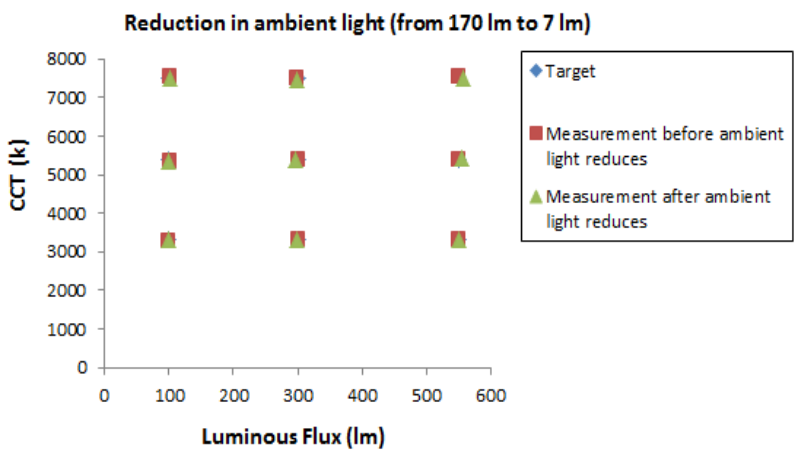

(b)

Fig. 20. Measured and target flux and CCT values of the bi-color LED lamp when (a) the ambient light intensity increases from $7 \mathrm{~lm}$ to $170 \mathrm{~lm}$ and (b) the ambient light intensity decreases from $170 \mathrm{~lm}$ to $7 \mathrm{~lm}$.

The experimental results show that despite the variations in ambient light intensity, the bi-color LED lamp with closed-loop nonlinear control remains stable in that the steady-state values of flux and CCT after the transition in ambient light are in close agreement with those before the change in ambient light. The maximum error in the steady-state measurements of luminous flux before and after the transient in ambient light is around 2.94\%. The maximum error in the steady-state measurements of CCT before and after the transient in ambient light is around $2.44 \%$. In addition, the maximum error between the measured flux value after transient and the target flux value is $2 \%$ and the maximum error between the measured CCT value after transient and the target CCT value is $2.64 \%$. Therefore, it is experimentally verified that the actual flux and CCT values continue to track closely with the target values in spite of the ambient light variations. In other words, it confirms the 
robustness of the proposed ambient light compensation scheme.

In reality, after illuminating an LED die for an extended period of time, the die itself will experience aging and degradation. For instance, the rated luminous flux of an LED light bulb is specified to be $500 \mathrm{~lm}$. After using it for one year, the actual luminous flux will drop to, say $470 \mathrm{~lm}$, and this aging effect will become permanent. Instead of performing accelerated aging tests, the effect of LED aging can be emulated by using another bi-color LED lamp whose average flux values are smaller than the original one. The main purpose is to demonstrate that the proposed nonlinear control scheme continues to produce consistent values between measured and target flux and CCT, regardless of LED aging. Fig. 21 shows the measured flux and CCT values between the new LED and the "aging" LED and their corresponding target values.

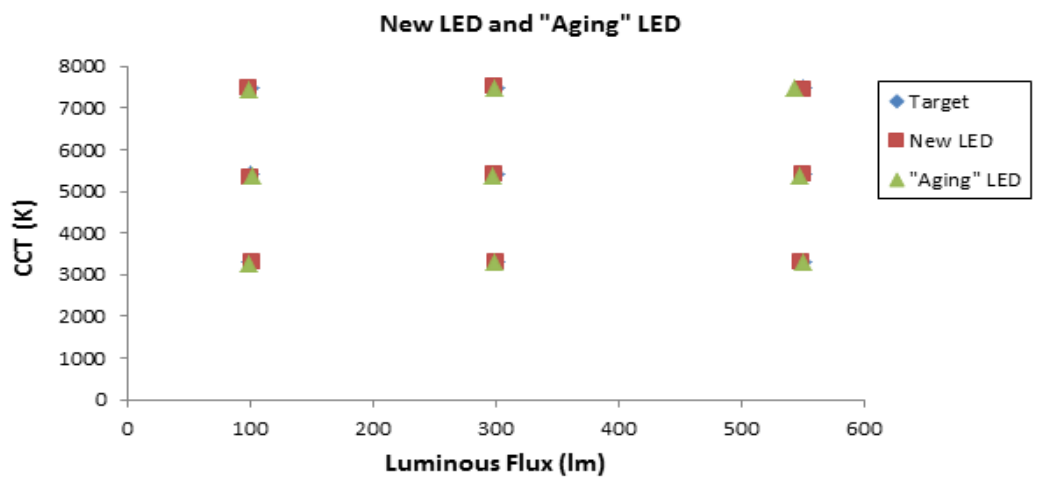

Fig. 21. Measured and target flux and CCT values between the new LED and the "aging" LED with respect to the corresponding target values.

The maximum error in luminous flux between the measured and target data for the new and "aging" LEDs are $1 \%$ and $2 \%$, respectively. The maximum error in CCT between the measured and target data for the new and "aging' LEDs are $1.15 \%$ and $0.91 \%$, respectively. Hence, it is experimentally verified that the proposed nonlinear feedback approach enables precise control between measured and target flux and CCT values despite LED aging.

\section{CONCLUSIONS}

A closed-loop nonlinear control scheme was proposed for the bi-color LED lamp to achieve precise and fullyindependent control of luminous flux and CCT under various operating conditions. The proposed control 
approach is based upon the nonlinear empirical models of LEDs with feedback control of flux and CCT using piecewise linear interpolation. Ambient light compensation is also employed to mitigate the effect of ambient light variations on the regulation of flux and CCT. It is experimentally verified that the proposed bi-color LED system using the closed-loop nonlinear control significantly improves the accuracy of light intensity and color control as compared with the existing bi-color lamps using closed-loop linear control. It is also demonstrated that the proposed LED system is robust to ambient temperature variations even without the use of a temperature sensor. The simplicity of the control algorithm, the reduction of hardware resources, and the generation of precise and consistent light output from the proposed bi-color LED system makes it an attractive solution for performing wide-range dimming and color-mixing in high-quality lighting applications.

\section{ACKNOWLEDGMENT}

This work was supported by the Hong Kong Research Grant Council under the Theme-base project T22-

715/12N. The authors are also grateful to the University of Hong Kong for its financial support for the patent application [32] for the method reported in this paper.

\section{REFERENCES}

[1] H. Chen, D. Y. Lin, S. C. Tan, and S. Y. Hui, "Chromatic, photometric and thermal modeling of LED systems with nonidentical LED devices", IEEE Trans. on Pow. Elect., vol. 29, no. 12. pp. 6636-6647, Dec. 2014.

[2] K. H. Loo, Y. M. Lai, S. C. Tan, and C. K. Tse, "On the color stability of phosphor-converted white LEDs under DC, PWM, and bilevel drive", IEEE Trans. on Power Elect., vol. 27, no. 2, pp. 974-984, Feb. 2012.

[3] F.-C. Wang, C.-W. Tang, B.-J. Huang, "Multivariable robust control for a red-green-blue LED lighting system", IEEE Trans. on Power Electronics, vol. 25, no. 2, pp.417-428, Feb. 2010.

[4] X. Qu, S. C. Wong and C. K. Tse, "Color control system for RGB LED light sources using junction temperature measurement”, in Proc. IEEE Industrial Electronics Society (IECON), pp. 1363- 1368, Nov. 5-8, 2007.

[5] S. Muthu, F. J. Schuurmans, and M. D. Pashley, "Red, green, and blue LED based white light generation: issues and control", Proc. Ind. Appl. Conf., pp. 327 - 333, Oct. 2002.

[6] M. Dyble, N. Narendran, A. Bierman, and T. Klein, "Impact of dimming white LEDs: chromaticity shifts due to different dimming methods", Proc. SPIE, vol. 5941, pp. 291-299, 2005.

[7] K. H. Loo, Y. M. Lai, S. C. Tan, and C. K. Tse, "Stationary and adaptive color-shift reduction methods based on the bilevel driving technique for phosphor-converted white LEDs", IEEE Trans. on Power Electronics, vol. 26, no. 7. pp. 1943-1953, Jul. 2011.

[8] M. M. Sisto, J. Gauvin, "Accurate chromatic control and color rendering optimization in LED lighting systems using junction temperature feedback", Proceedings of SPIE, vol. 9190, Sep. 2014.

[9] A. Lee, J. Sin, P. Chan, "Scalability of quasi-hysteretic FSM-based digitally controlled single-inductor dualstring buck LED driver to multiple strings", IEEE Trans. on Pow. Elect., pp. 501-513, vol. 29, no. 1, Jan. 2014.

[10] H. C. Kim, C. S. Yoon, H. Ju, D.-K. Jeong, J. Kim, “An AC-powered, flicker-free, multi-channel LED driver with current-balancing SIMO buck topology for large area lighting applications", in IEEE APEC, pp. 3337-3341, Mar. 2014. 
[11] H. Chen, Y. Zhang, and D. Ma, "A SIMO parallel-string driver IC for dimmable LED backlighting with local bus voltage optimization and single time-shared regulation loop", IEEE Trans. on Power Electronics, vol. 27, no. 1, pp. 452-462, Jan. 2012.

[12] W. Zhang, P. Sutardja, "Correlated color temperature control methods and devices", U.S. Patent US20130020956 A1, Jan. 24, 2013.

[13] Peng Liu, et al. "Investigation of self-adaptive LED surgical lighting based on entropy contrast enhancing method", Optics Communication, vol. 319, pp. 133-140, May 2014.

[14] H.-C. Wang, et al. "Enhanced visualization of oral cavity for early inflamed tissue detection", Optics Express, vol. 18, no. 11, pp. 11800-11809, May 2010.

[15] H.-C. Wang, et al. "Optimal lighting of RGB LEDs for oral cavity detection”, Optics Express, vol. 20, no. 9, pp. 10186-10199, Apr. 2012.

[16] H.-C. Wang, et al. "Anti-glare LED lamps with adjustable illumination light field", Optics Express, vol. 22 , no. 5, Mar. 2014.

[17] D. Ramane and A. Shaligram, "Optimization of multi-element LED source for uniform illumination of plane surface”, Optics Express, vol. 19, no. s4, pp. A639-A648, Jul. 2011.

[18] D. Corell, et al. "Light Emitting Diodes as an alternative ambient illumination source in photolithography environment", vol. 17, no. 20, pp. 17293-17302, Sep. 2009.

[19] H.-T. Chen, et al. "Nonlinear Dimming and Correlated Color Temperature Control of Bi-Color White LED Systems", IEEE Trans. on Power Electronics, (early access).

[20] O. Noboru, Colorimetry: fundamentals and applications, John Wiley \& Sons, 2006.

[21] Y. Gu, N. Narendran, T. Dong, and H. Wu, "Spectral and luminous efficacy change of high-power LEDs under different dimming methods", Proceedings of SPIE, 2006.

[22] J. Garcia, M. Dalla-Costa, J. Cardesin, J. Alonso, and M. Rico-Secades, "Dimming of high-brightness LEDs by means of luminous flux thermal estimation", IEEE Trans. on Pow. Elect., vol. 24, no. 4, pp. 1107-1114, Apr. 2009.

[23] K. Man and I. Ashdown, "Accurate colorimeter feedback for RGB LED clusters", Proceedings of SPIE., vol. 6337, 2006.

[24] J. Smith, "Calculating color temperature and illuminance using the TAOS TCS3414CS digital color sensor”, Designer's Notebook, pp. 1-7, Feb. 2009 [Online], Available: https://www.ams.com/ger/content/view/download/145158

[25] American National Standard, Specifications for Chromaticity of Solid State Lighting Products, ANSI C78.377 (American National Standards Institute, 2008).

[26] User's Manual: PMS-50/80, "PMS-50/80 UV-VIS-near IR Spectrophotocolorimeter", version 3.0, Everfine Photo-E-Info Co., Ltd.

[27] Datasheet: TCS3472, "Color light-to-digital converter with IR filter", Texas Advanced Optoelectronic Solutions (TAOS), [Online], Available: http://ams.com/chi/content/download/319364/1117183/file/TCS3472_Datasheet_EN_v1.pdf

[28] G. Sharma and H. J. Trussell, "Digital color imaging", IEEE Trans. on Image Processing, vol. 6, no. 7, pp. 901-932, Jul. 1997.

[29] Datasheet: XLamp XP-E LED, "Cree XLamp XP-E Product Family Data sheet", Cree Inc., [Online], Available: http://www.cree.com/ /media/Files/Cree/LED\%20Components\%20and\%20Modules/XLamp/Data\%20and\%2 0Binning/XLampXPE.pdf

[30] Datasheet: ECL10US48, "5-30 watts ECL series", XP Power Ltd., [Online], Available: http://www.xppower.com/pdfs/SF_ECL05-30.pdf

[31] Datasheet: LM3402HV, "0.5A constant current buck regulator for driving high power LEDs", Texas Instruments Inc., [Online], Available: http://www.ti.com/lit/ds/symlink/lm3402.pdf

[32] H. T. Chen, S. C. Tan, and S. Y. R. Hui, "Correlated color temperature control system and method," PCT Application: PCT/CN2014/078552, May 2014. 